\title{
Candidate free-floating super-Jupiters in the young $\sigma$ Orionis open cluster
}

\author{
G. Bihain ${ }^{1,2}$, R. Rebolo ${ }^{1,2}$, M. R. Zapatero Osorio ${ }^{1}$, V. J. S. Béjar ${ }^{1}$, I. Villó-Pérez ${ }^{3}$, A. Díaz-Sánchez ${ }^{3}$, \\ A. Pérez-Garrido ${ }^{3}$, J. A. Caballero ${ }^{4}$, C. A. L. Bailer-Jones ${ }^{5}$, D. Barrado y Navascués ${ }^{6,7}$, J. Eislöffel ${ }^{8}$, T. Forveille ${ }^{9}$, \\ B. Goldman ${ }^{5}$, T. Henning 5 , E. L. Martín ${ }^{1,10}$, and R. Mundt ${ }^{5}$ \\ 1 Instituto de Astrofísica de Canarias, c/ vía Láctea, s/n, 38205 La Laguna, Tenerife, Islas Canarias, Spain \\ e-mail: gbihain@ll.iac.es \\ 2 Consejo Superior de Investigaciones Científicas, Spain \\ 3 Universidad Politécnica de Cartagena, Campus Muralla del Mar, 30202 Cartagena, Murcia, Spain \\ ${ }^{4}$ Dpto. de Astrofísica y Ciencias de la Atmósfera, Facultad de Física, Universidad Complutense de Madrid, 28040 Madrid, Spain \\ 5 Max-Planck-Institut für Astronomie, Königstuhl 17, 69117 Heidelberg, Germany \\ ${ }^{6}$ Laboratorio de Astrofísica Espacial y Exoplanetas, Centro de Astrobiologia (LAEFF-CAB, INTA-CSIC), \\ European Space Astronomy centre (ESAC), PO Box 78, 28691 Villanueva de la Cañada, Madrid, Spain \\ 7 Spanish Virtual Observatory thematic network, Spain \\ 8 Thüringer Landessternwarte Tautenburg, Sternwarte 5, 07778 Tautenburg, Germany \\ 9 Laboratoire d'Astrophysique de Grenoble, Observatoire de Grenoble, Université Joseph Fourier, CNRS, UMR 571, Grenoble, \\ France \\ 10 University of Central Florida. Department of Physics, PO Box 162385, Orlando, FL 32816-2385, USA \\ Received 26 March 2009 / Accepted 31 July 2009
}

\section{ABSTRACT}

Context. Free-floating substellar candidates with estimated theoretical masses of as low as $\sim 5$ Jupiter masses have been found in the $\sim 3 \mathrm{Myr}$ old $\sigma$ Orionis open cluster. As the overlap with the planetary mass domain increases, the question of how these objects form becomes important. The determination of their number density and whether a mass cut-off limit exists is crucial to understanding their formation.

Aims. We propose to search for objects of yet lower masses in the cluster and determine the shape of the mass function at low mass. Methods. Using new- and (re-analysed) published $I Z J H K_{\mathrm{s}}[3.6]-[8.0]$-band data of an area of $840 \mathrm{arcmin}^{2}$, we performed a search for LT-type cluster member candidates in the magnitude range $J=19.5-21.5 \mathrm{mag}$, based on their expected magnitudes and colours. Results. Besides recovering the T type object S Ori 70 and two other known objects, we find three new cluster member candidates, S Ori 72-74, with $J \approx 21 \mathrm{mag}$ and within $12 \operatorname{arcmin}$ of the cluster centre. They have theoretical masses of $4_{-2}^{+3} M_{\text {Jup }}$ and are among the least massive free-floating objects detected by direct imaging outside the Solar System. The photometry in archival Spitzer [3.6]-[5.8]-band images infers that S Ori 72 is an L/T transition candidate and S Ori 73 a T-type candidate, following the expected cluster sequence in the mid-infrared. Finally, the L-type candidate S Ori 74 with lower quality photometry is located at 11.8 arcsec $(\sim 4250 \mathrm{AU})$ of a stellar member of $\sigma$ Orionis and could be a companion. After contaminant correction in the area complete to $J=21.1 \mathrm{mag}$, we estimate that there remain between zero and two cluster members in the mass interval 6-4 $M_{\text {Jup }}$.

Conclusions. We present S Ori 73, a new candidate $\mathrm{T}$ type and candidate $\sigma$ Orionis member of a few Jupiter masses. Our result suggests a possible turnover in the substellar mass spectrum below $\sim 6$ Jupiter masses, which could be investigated further by wider and deeper photometric surveys.

Key words. stars: luminosity function, mass function - Galaxy: open clusters and associations: individual: $\sigma$ Orionis stars: low-mass, brown dwarfs

\section{Introduction}

Free-floating objects with masses of several to a few times the mass of Jupiter appear to populate young open clusters (see Lucas \& Roche 2000; Zapatero Osorio et al. 2000). They could form in a similar way to stars, by gravitational fragmentation above an opacity mass limit (Hoyle 1953; Larson 1973; Low \& Lynden-Bell 1976; Rees 1976; Silk 1977) or by turbulent fragmentation (Padoan \& Nordlund 2002, 2004; Padoan et al. 2007) of collapsing molecular clouds, or as stellar embryos that are fragmented, photo-eroded, or ejected before they can accrete sufficient mass to become stars (see Whitworth \& Goodwin 2005, and references therein). They could also form by gravitational instability in circumstellar disks (Boss 1997; Whitworth \& Stamatellos 2006), then have their orbits disrupted and be ejected (Stamatellos \& Whitworth 2009; Veras et al. 2009). A better knowledge of the cluster mass function (MF; number of objects per unit mass) at these low masses will help us to determine the main formation process for these objects. Indeed, numerical simulations of opacity-limited fragmentation show a cutoff in the mass function at $\sim 4$ Jupiter masses (Bate \& Bonnell 2005; Bate 2005, 2009), whereas numerical simulations of turbulent fragmentation show an approximately log-normal, shallower drop at substellar masses (Padoan \& Nordlund 2004). A detailed comparison with planets (e.g., spectral emission and chemical composition) will also provide complementary information about their origin and evolution (Fortney et al. 2008).

The $\sigma$ Orionis open cluster in the Ori OB $1 \mathrm{~b}$ association, together with other star-forming regions in the Orion and 
Scorpius-Centaurus complexes, is well-suited to the search for free-floating planetary-mass objects. It is young $(3 \pm 2 \mathrm{Myr}$; Zapatero Osorio et al. 2002b), relatively nearby $\left(360_{-60}^{+70} \mathrm{pc}\right.$, Brown et al. 1994; $444 \pm 20$ pc, Sherry et al. 2008), affected by very low extinction $\left(A_{\mathrm{V}}<1 \mathrm{mag}\right.$; Sherry et al. 2008), and of solar metallicity (González Hernández et al. 2008). A revision of published, basic parameters of the cluster was provided by Caballero (2007). Caballero et al. (2007) found a smoothly continuous MF down to $\sim 6$ Jupiter masses $\left(M_{\text {Jup }}\right)$ and that the brown dwarfs appear to harbour disks with a frequency similar to that of low-mass stars. This suggests that low-mass stars and substellar objects share the same formation mechanism. Also, S Ori 70, of spectral type T6, has been proposed to be a cluster member with an estimated mass of 2-7 $M_{\text {Jup }}$ (Zapatero Osorio et al. 2002a, 2008; Martín \& Zapatero Osorio 2003; Burgasser et al. 2004; Scholz \& Jayawardhana 2008; Luhman et al. 2008).

In this paper, we present new $I Z J H K_{\mathrm{s}}$-band photometry and a re-analysis of previous data of the $\sigma$ Orionis cluster, allowing us to search for faint candidates in an area of $\sim 790 \mathrm{arcmin}^{2}$, to the completeness magnitude $J \approx 21.1 \mathrm{mag}$. Our search area overlaps with those of Caballero et al. (2007) and Lodieu et al. (2009b), and its $J$-band completeness magnitude is about 1.5 and 2 mag fainter, respectively. We report the detection of three new cluster member candidates with theoretical masses of $\sim 4 M_{\text {Jup }}$.

\section{Observations and data reduction}

We discuss the new data obtained for this study and the data from Caballero et al. (2007) and Zapatero Osorio et al. (2008) that were reduced or analysed again in an attempt to increase the sensitivity to faint sources.

\subsection{Optical data}

The $I$-band imaging data presented in Caballero et al. (2007) were obtained with the Wide Field Camera (WFC) mounted at the Isaac Newton Telescope (INT). The WFC contains four CCD of $2 \mathrm{k} \times 4 \mathrm{k}$ pixels and 0.33 arcsec/pixel. Figure 2 shows the area of the corresponding four images, limited to their overlap with the near-infrared data (solid and dashed lines). A new automatic search for sources was performed with the IRAF routine FINDSTAR (Almoznino), which led to a substantial increase in the number of sources at faint magnitudes with respect to those considered by Caballero et al. (2007). FINDSTAR is especially useful for detecting sources in combined dithered images (or in images with background gradients), where the standard deviation varies from centre to border. We then carried out the aperture and point-spread-function (PSF) photometry using routines within the DAOPHOT package. Objects missed by the automatic search routine but easily detected by eye in the PSF-subtracted images (e.g., sources partially hidden in the wings of bright stars) were added to the list of sources. Finally, for each of the four CCD images, the new photometry was calibrated using $\sim 1850$ objects in common with the Caballero et al. (2007) photometry in the Cousins system. We found average completeness and limiting magnitudes of $I_{\mathrm{cmp}}=23.0 \mathrm{mag}$ and $I_{\mathrm{lim}}=23.9 \mathrm{mag}$, respectively.

To determine these completeness and limiting magnitudes, we compiled the distribution of the instrumental magnitude error versus the calibrated magnitude for each image. In the bottom panel of Fig. 1, we present this with the source catalogue of one of the WFC CCD images. The completeness and limiting magnitudes were defined to be the faintest magnitude bins where the average errors are $\leq 0.10$ and $0.20 \mathrm{mag}$, respectively.
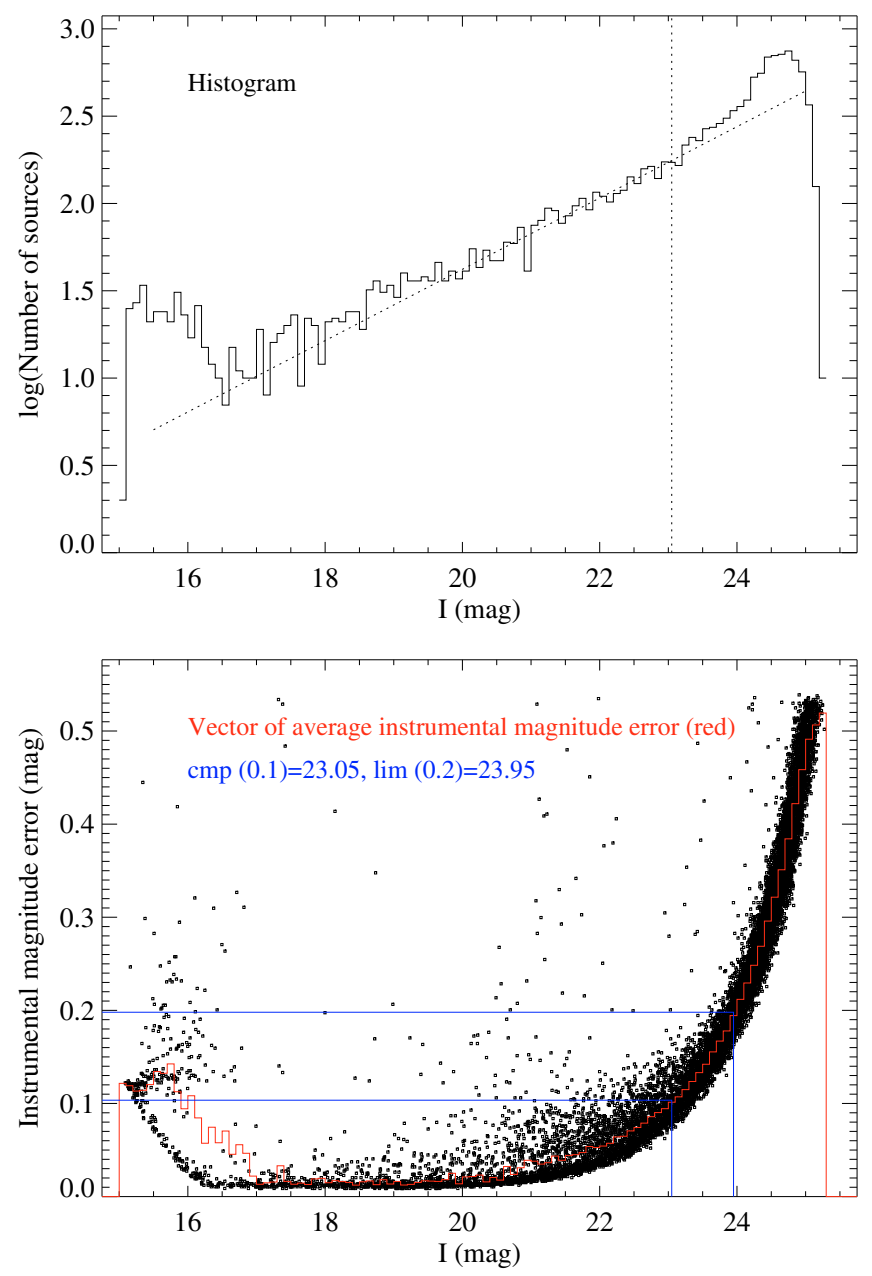

Fig. 1. Upper panel. Histogram in logarithmic scale of the $I$-band sources as a function of the calibrated magnitude (see text for details about the dotted lines). Lower panel. Instrumental magnitude error versus the calibrated magnitude of these sources (dots). The average error per magnitude bin is overploted as a red solid line. The magnitudes of the bins just below errors of 0.1 mag (blue lower line) and $0.2 \mathrm{mag}$ (blue upper line) were defined here as the completeness and limiting magnitudes, respectively.

These errors correspond to signal-to-noise ratios of $S / N=10$ and $S / N=5$, respectively (see e.g., Newberry 1991). The average error per magnitude bin is overploted as a red solid line. The upper panel shows the histogram of all sources on a logarithmic scale as a function of the calibrated magnitude. The inclined dotted line represents a power law fit to the histogram in the range $\left[\mathrm{mag}_{\mathrm{cmp}}-2.5, \mathrm{mag}_{\mathrm{cmp}}\right]$, where $\operatorname{mag}_{\mathrm{cmp}}$ is the completeness magnitude (vertical dotted line). At mag $>$ mag $_{\mathrm{cmp}}$, the histogram's deviation from the fit is most probably caused by sources affected by random upward or downward fluctuations of the background, the upward ones being preferentially detected above the detection threshold (Malmquist bias; see also Beichman et al. 2003). Comparing the counts of the histogram of sources having errors smaller than 0.10 mag with the counts of the linear extrapolation of its power law fit at the completeness magnitude, we estimated a level of completeness of $\gtrsim 90 \%$. This method was also applied to the other data sets.

We used broad IZ-band images from the Keck II Low Resolution Imaging Spectrograph (LRIS), associated with the discovery of S Ori 70 (see Zapatero Osorio et al. 2002a), as well as unpublished $I$-band images obtained with the same 


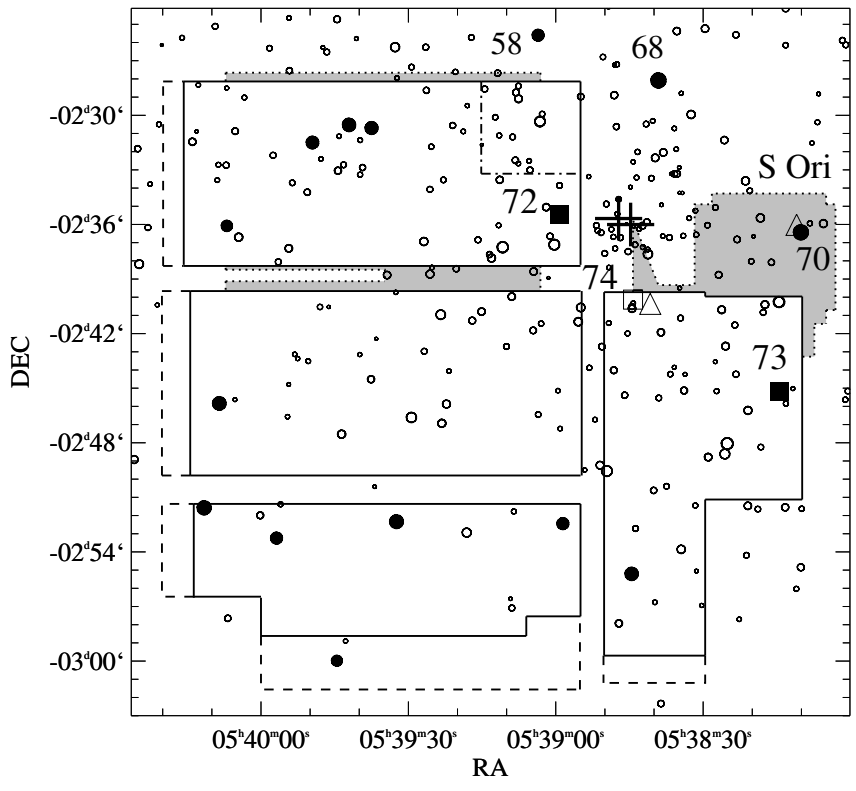

Fig. 2. Main search area: WFC-ISAAC $I J$-band data (dashed line) together with follow-up $H$ - or $K$-band data (solid line). Additional search areas: WFC $I$-, LRIS $I$-, and Omega2000 $J H K_{\mathrm{s}}$-band data (shaded regions delimited by dotted lines). Individual fields are not represented for clarity (see Fig. A.1). These areas have a completeness $J \geq 21.1 \mathrm{mag}$, except the upper corner in the main area (indicated by the dash-dot line) and the shaded regions on the left side. S Ori 72 and S Ori 73 are represented by filled squares, S Ori 74 by an open square, and S Ori J053840.8-024022 and S Ori J053811.0-023601 by open triangles (see finding charts of Figs. B.1-B.4). Circular symbols are cluster members and candidates (Zapatero Osorio et al. 2000; Caballero et al. 2007; Caballero 2008b); their size increases with fainter J-band magnitude, to highlight the location of the least massive objects. Filled circles are planetary-mass candidates with $M<0.013 M_{\odot}$, including S Ori 70 (Zapatero Osorio et al. 2002a). Crosses are $\sigma$ Ori AB and E, at the centre of the cluster.

instrument on 2000 January 5, in similar atmospheric conditions and for the same exposure time. Their coordinates and depths are listed in Table 1 . The photometry was performed as described above. The $I$-band photometry was calibrated using typically 200 sources in common with the WFC survey, whereas the Z-band photometry was calibrated using on average 41 stellar sources from the Galactic Clusters Survey (GCS) component of UKIDSS $^{1}$ (Lawrence et al. 2007, fifth data release) and of magnitude error $\sigma_{Z}$ (UKIDSS) $<0.1 \mathrm{mag}$. The $Z$-band photometry has an average (relative) calibration error of 0.04 mag. We caution that the UKIDSS Z-band filter differs from that of the LRIS images. For the published data, we estimated that the average completeness and limiting magnitudes are $I_{\mathrm{cmp}}=23.5 \mathrm{mag}$ and $I_{\text {lim }}=24.3 \mathrm{mag}$, respectively, and $Z_{\mathrm{cmp}}=22.3 \mathrm{mag}$ and $Z_{\text {lim }}=23.2 \mathrm{mag}$, respectively. For the data from 2000, these are $I_{\mathrm{cmp}}=23.5 \mathrm{mag}$ and $I_{\text {lim }}=24.5 \mathrm{mag}$.

We obtained Z-band imaging data using the INT/WFC instrument on the night of 2008 November 27. We took 21 images of $900 \mathrm{~s}$ exposure time each and with central coordinates $(\alpha, \delta)=(53749.1,-24443)$. During the observations, thin cirrus were present and the average seeing was 1.2 arcsec. The images were reduced using routines within the IRAF

1 UKIDSS uses the UKIRT Wide Field Camera (WFCAM, Casali et al. 2007) and a photometric system described in Hewett et al. (2006). The pipeline processing and science archive are described in Hambly et al. (2008).
Table 1. Coordinates and depth of the LRIS images ${ }^{a}$.

\begin{tabular}{ccccc}
\hline \hline ID & $\begin{array}{c}\alpha \\
(\mathrm{J} 2000)\end{array}$ & $\begin{array}{c}\delta \\
(\mathrm{J} 2000)\end{array}$ & Filter & $\begin{array}{c}m_{\text {cmp }}, m_{\text {lim }} \\
(\mathrm{mag})\end{array}$ \\
\hline $1998 \mathrm{i} 1$ & 053816.6 & -023753 & $I$ & $23.6,24.5$ \\
$1998 \mathrm{i} 2$ & 053821.0 & -024759 & $I$ & $23.5,24.4$ \\
$1998 \mathrm{3} 3$ & 053856.4 & -023857 & $I$ & $23.5,24.1$ \\
$1998 \mathrm{i} 4$ & 053921.2 & -023754 & $I$ & $23.5,24.4$ \\
$1998 \mathrm{5} 5$ & 053956.4 & -023400 & $I$ & $23.6,24.4$ \\
$1998 \mathrm{z} 1$ & 053816.6 & -023753 & $Z$ & $22.1,22.9$ \\
$1998 \mathrm{z} 2$ & 053821.0 & -024759 & $Z$ & $22.4,23.3$ \\
$1998 \mathrm{z} 3$ & 053856.4 & -023857 & $Z$ & $22.4,23.3$ \\
$1998 \mathrm{z} 4$ & 053921.2 & -023754 & $Z$ & $22.5,23.4$ \\
$1998 z 5$ & 053956.4 & -023400 & $Z$ & $22.4,23.3$ \\
$2000 \mathrm{i} 1$ & 053817.4 & -023748 & $I$ & $23.8,24.6$ \\
$2000 \mathrm{i} 2$ & 053821.4 & -024803 & $I$ & $23.5,24.5$ \\
$2000 i 3$ & 053855.2 & -023852 & $I$ & $23.3,24.3$ \\
$2000 \mathrm{i} 4$ & 053920.3 & -023758 & $I$ & $23.7,24.5$ \\
$2000 \mathrm{5} 5$ & 053957.2 & -023404 & $I$ & $23.6,24.7$ \\
\hline
\end{tabular}

${ }^{a}$ The pixel scale is 0.210 arcsec pixel $^{-1}$ and the field of view in the $\alpha-\delta$ frame is $5.8 \times 7.2 \sim \operatorname{arcmin}^{2}$. For the images $1998[\mathrm{i}, \mathrm{z}] 3,2000 \mathrm{i}[1$, $2,4,5]$, and 2000i3, this field of view is rotated $\sim 16,90$, and $105 \mathrm{deg}$ counterclockwise, respectively.

environment, including bias and zero image subtraction and flatfield correction. Observations were done using a dithering pattern. Science images were combined to obtain flat-field images to correct for fringing. Individual images were aligned and combined to obtain final images. Aperture and PSF photometry was performed for one of the CCDs. Its photometric calibration was done using about 400 stellar sources from GCS-UKIDSS of $\sigma_{Z \text { (UKIDSS) }}<0.1 \mathrm{mag}$, implying a relative calibration error of $0.02 \mathrm{mag}$. We caution that the UKIDSS $Z$-band filter is different from that of the WFC images. We estimated completeness and limiting magnitudes of 22.4 and $23.1 \mathrm{mag}$, respectively.

Astrometry was obtained for all the optical images with an accuracy of $\sim 0.2-0.05$ arcsec, using 2MASS as reference and an adaptation of the IRAF MYASTROM procedure (Puddu, see also Bihain et al. 2006). A representation of the individual fields is provided in Fig. A.1 (top left and right panels).

\subsection{Near- and mid-infrared data}

The J-band imaging data from Caballero et al. (2007) were obtained with the Infrared Spectrometer And Array Camera (ISAAC), mounted at the Very Large Telescope (VLT) and containing a Rockwell Hawaii detector of $1 \mathrm{k} \times 1 \mathrm{k}$ pixels and $0.148 \mathrm{arcsec} /$ pixel. We re-reduced these data to obtain a clean sky subtraction, remove bad pixel values, and identify more reliably charge persistencies of bright sources in the detector. The raw images were dark subtracted, superflat divided, sky subtracted (with the routine LIRISDR. LIMAGE. LRUNSKY from Acosta-Pulido, which includes object masking and vertical gradient correction), their elements flagged for bad pixel (at extreme values for thresholding) using bad pixel masks from superflats, related by pixel shifts (computed from clearly defined sources in common), and combined all at once in strips along right ascension or declination (16 strips in total). The photometry of each strip was performed similarly as for the WFC data. We ensured that all the sources remaining in the PSF-subtracted images were recovered, as we did for the Omega2000 J-band images (see below). The photometry was calibrated using an average number of 12 point sources from the 2 MASS catalogue (Skrutskie et al. 2006) of quality flags AAA or AAB. The average calibration 
Table 2. New near-infrared observations ${ }^{a}$.

\begin{tabular}{|c|c|c|c|c|c|c|c|c|c|}
\hline ID & $\begin{array}{c}\alpha \\
(\mathrm{J} 2000)\end{array}$ & $\begin{array}{c}\delta \\
(\mathrm{J} 2000) \\
\end{array}$ & Instr. & Filter & $\begin{array}{c}\text { Area } \\
\left(\operatorname{arcmin}^{2}\right)\end{array}$ & Date(s) & $\begin{array}{c}t_{\exp } \\
(\mathrm{min})\end{array}$ & $\begin{array}{l}m_{\mathrm{cmp}} \\
(\mathrm{mag})\end{array}$ & $\begin{array}{c}m_{\lim } \\
(\mathrm{mag})\end{array}$ \\
\hline $1-\mathrm{k}$ & 053939.9 & -025026 & Omega2000 & $K_{\mathrm{s}}$ & 197 (279) & 2005 Jan. 31, Feb. 1, Oct. 26 & 326 & 20.5 & 21.2 \\
\hline $2-j$ & 053935.1 & -023444 & Omega2000 & $J$ & $214(259)$ & 2006 Oct. 07 & 63 & 20.9 & 21.9 \\
\hline 2-h & 053919.3 & -023336 & Omega2000 & $H$ & $181(295)$ & 2005 Oct. 19 & 186 & 21.2 & 21.9 \\
\hline $2-\mathrm{k}$ & 053921.2 & -023514 & Omega2000 & $K_{\mathrm{s}}$ & $194(282)$ & 2005 Oct. $24-25$ & 139.5 & 20.1 & 21.0 \\
\hline 3-h & 053840.1 & -024809 & SofI & $H$ & $21(27)$ & 2006 Dec. 27 & 147 & 20.3 & 20.9 \\
\hline 4-h & 053840.0 & -025226 & SofI & $H$ & $21(27)$ & 2006 Dec. 24 & 98 & 20.3 & 21.1 \\
\hline $5-\mathrm{h}$ & 053839.7 & -025709 & SofI & $H$ & $21(27)$ & 2006 Dec. 27 & 75 & 20.1 & 20.5 \\
\hline 6-h & 053820.0 & -024830 & SofI & $H$ & $21(27)$ & 2006 Dec. 24 & 201 & 20.1 & 21.1 \\
\hline 7-h & 054005.1 & -023040 & SofI & $H$ & $21(27)$ & 2006 Dec. 27 & 132 & 20.3 & 21.1 \\
\hline $8-\mathrm{h}$ & 054004.9 & -023557 & SofI & $H$ & $21(27)$ & 2006 Dec. 26 & 98 & 19.5 & 20.1 \\
\hline 9-h & 054003.6 & -024210 & SofI & $H$ & $21(27)$ & 2006 Dec. 25 & 56 & 19.9 & 20.7 \\
\hline 10-h & 053909.7 & -024715 & SofI & $H$ & $21(27)$ & 2006 Dec. 25 & 168 & 20.3 & 21.3 \\
\hline 11-h & 053927.7 & -024715 & SofI & $H$ & $21(27)$ & 2006 Dec. 25 & 95 & 20.1 & 21.1 \\
\hline $12-\mathrm{h}$ & 053945.7 & -024715 & SofI & $H$ & $21(27)$ & 2006 Dec. 26 & 120 & 20.3 & 21.3 \\
\hline 13-h & 054003.1 & -024704 & SofI & $H$ & $16(33)$ & 2006 Dec. 26 & 140 & 20.3 & 21.1 \\
\hline 14-h & 053909.5 & -025356 & LIRIS & $H$ & $16(20)$ & 2006 Dec. 29 & 36 & 19.5 & 20.1 \\
\hline $15-\mathrm{h}$ & 053945.4 & -025357 & LIRIS & $H$ & $16(20)$ & 2007 Dec. 14 & 45 & 20.3 & 20.9 \\
\hline $16-\mathrm{h}$ & 054003.6 & -025359 & LIRIS & $H$ & $16(20)$ & 2007 Dec. 14 & 45 & 20.3 & 20.9 \\
\hline
\end{tabular}

${ }^{a}$ Fields of view (and pixel scales) of the Omega2000, SofI, and LIRIS detectors are $15.4 \times 15.4 \operatorname{arcmin}^{2}\left(0.45 \operatorname{arcsec}_{\operatorname{pixel}}^{-1}\right), 4.9 \times 4.9 \operatorname{arcmin}^{2}$ $\left(0.288 \operatorname{arcsec} \operatorname{pixel}^{-1}\right)$, and $4.2 \times 4.2 \operatorname{arcmin}^{2}\left(0.25 \operatorname{arcsec}\right.$ pixel $\left.^{-1}\right)$, respectively.

error is 0.03 mag. The average completeness and limiting magnitudes are $J_{\mathrm{cmp}}=21.6 \mathrm{mag}$ and $J_{\mathrm{lim}}=22.4 \mathrm{mag}$, respectively, in an area of $\sim 660 \mathrm{arcmin}^{2}$. This area excludes $\sim 20 \mathrm{arcmin}^{2}$ within the region delimited by the dash dot line in Fig. 2, where $J_{\text {cmp }}=20.6 \mathrm{mag}$ and $J_{\text {lim }}=21.5 \mathrm{mag}$, and $\sim 100 \mathrm{arcmin}^{2}$ corresponding to the $\sim 0.4 \mathrm{mag}$ shallower borders of the strips.

We obtained additional near-infrared imaging data, using Omega2000 at the 3.5-m Telescope (Calar Alto, Spain), Son of Isaac (SofI) at the New Technology Telescope (La Silla, Chile), and the Long-slit Intermediate Resolution Infrared Spectrograph (LIRIS) at the William Herschel Telescope (Roque de los Muchachos Observatory, Spain). Table 2 indicates field identification, coordinates, instrument, filter, area, observing night date(s), total exposure time, and completeness- and limiting magnitudes. All the data were reduced within the IRAF environment, including (super)flat division, sky subtraction, alignment with several reference stars, and combination without trimming. SofI raw images were first row cross-talk corrected with the routine crosstalk (Leo Vanzi, ESO SofI tools). Omega2000 and most of the SofI images were dark subtracted before flat division. LIRIS raw images were first pixel-mappingand row-cross-talk corrected, and then processed with the routine LIRISDR. LIMAGE. LDEDITHER, including sky subtraction, as applied to ISAAC $J$-band, and distortion correction. Bad pixel masks were used for LIRIS and SofI, whereas for Omega2000 extreme values relative to the average at each pixel were rejected during combination. Because the combined images are untrimmed, they have a deep central region surrounded by a shallower region, whose proportions depend on the observing dithers. In Table 2, we list the deep area and the total area (in parenthesis). The photometry was obtained as described above. For the Omega2000 images, the photometric calibration was obtained using 150 2MASS point sources of quality flags AAA or $\mathrm{AAB}$ (average calibration error of $0.03 \mathrm{mag}$ ). For each of the SofI and LIRIS images, about 10 of these calibrators were used (average calibration error of $0.04 \mathrm{mag}$ ). Completeness and limiting magnitudes were derived from the sources in the deeper central regions.
Table 3. Re-estimated depth of individual fields from Caballero et al. (2007) and Zapatero Osorio et al. (2008).

\begin{tabular}{cccccc}
\hline \hline ID & $\begin{array}{c}\alpha \\
(\mathrm{J} 2000)\end{array}$ & $\begin{array}{c}\delta \\
(\mathrm{J} 2000)\end{array}$ & Instr. & Filter & $\begin{array}{c}m_{\text {cmp }}, m_{\text {lim }} \\
(\mathrm{mag})\end{array}$ \\
\hline $1-\mathrm{h}$ & 053937.6 & -024953 & Omega2000 & $H$ & $20.0,20.9$ \\
$17-\mathrm{h}$ & 053838.7 & -024901 & Omega2000 & $H$ & $19.1,19.6$ \\
$17-\mathrm{k}$ & 053837.6 & -024951 & Omega2000 & $K_{\mathrm{s}}$ & $19.6,20.3$ \\
$18-\mathrm{j}{ }^{a}$ & 053812.4 & -023518 & Omega2000 & $J$ & $21.1,21.9$ \\
$18-\mathrm{h}^{a}$ & 053811.9 & -023459 & Omega2000 & $H$ & $20.6,21.4$ \\
$18-\mathrm{k}^{a}$ & 053813.3 & -023537 & Omega2000 & $K_{\mathrm{s}}$ & $20.0,21.0$ \\
$19-\mathrm{h}$ & 053844.6 & -024435 & CFHTIR & $H$ & $21.1,22.1$ \\
$19-\mathrm{k}$ & 053844.6 & -024435 & CFHTIR & $K^{\prime}$ & $20.9,21.9$ \\
$20-\mathrm{h}$ & 053818.3 & -024431 & CFHTIR & $H$ & $20.5,21.3$ \\
$20-\mathrm{k}$ & 053818.3 & -024431 & CFHTIR & $K^{\prime}$ & $20.5,21.3$ \\
$21-\mathrm{h}$ & 053924.2 & -022936 & CFHTIR & $H$ & $20.9,21.5$ \\
$21-\mathrm{k}$ & 053925.6 & -022937 & CFHTIR & $K^{\prime}$ & $20.5,21.3$ \\
$22-\mathrm{h}$ & 054006.5 & -023229 & CFHTIR & $H$ & $20.7,21.9$ \\
$22-\mathrm{k}$ & 054006.6 & -023229 & CFHTIR & $K^{\prime}$ & $20.5,21.5$ \\
\hline
\end{tabular}

${ }^{a}$ Re-reduced data from Zapatero Osorio et al. (2008); deep central areas (and total areas) in $J, H$, and $K_{\mathrm{s}}$ are 215 (257), 208 (265), and 197 (278) $\mathrm{arcmin}^{2}$. For the Omega2000 and CFHTIR data from Caballero et al. (2007), the deep central areas (and total areas) are 216 (236) and 6 (22) $\operatorname{arcmin}^{2}$, respectively.

Other near-infrared data, already published in Caballero et al. (2007) and Zapatero Osorio et al. (2008), were used in the search. The Omega2000 data from the latter study were re-reduced to obtain untrimmed images. Completeness and limiting magnitudes of our new photometry (obtained as described above) are listed in Table 3, except for the $H$-band 1100 $\mathrm{arcmin}^{2}$ Omega2000 survey from 2003 (see Caballero et al. 2007), which is shallower. We note that the field $1-\mathrm{k}$ is a combination of new data obtained on 2005 October 26 with published data obtained earlier the same year, on January 31 and February 1 . The $J$ - and $H K$-band data from Tables 2 and 3 correspond to overlapping areas of $\sim 240 \mathrm{arcmin}^{2}$ and $\sim 690 \mathrm{arcmin}^{2}$ 
with the WFC + ISAAC survey, respectively (see Fig. 2 for the $H K$-band data).

Astrometry was obtained for all the near-infrared images similarly as for the optical images, with an accuracy of $\sim 0.2-0.05$ arcsec. A representation of the individual fields is provided in Fig. A.1 (top left and bottom panels).

We also used archival post-basic calibrated data (PBCD) from the Spitzer Space Telescope Infrared Array Camera (IRAC). For our new candidates (see Sect. 4), we have obtained the Spitzer photometry following the procedure described in Zapatero Osorio et al. (2007) and using the data published by Hernández et al. (2007) and Scholz \& Jayawardhana (2008). A comparison of these two data sets is provided in Luhman et al. (2008, see e.g. Fig. 1 therein for a map of the IRAC surveys). We averaged our measurements in overlapping deep images and adopted their standard deviation as a representative error bar. We compared the [3.6]- and [4.5]-band measurements of Zapatero Osorio et al. (2007) with those of Luhman et al. (2008) for the six objects in common ${ }^{2}$ and found small average differences $[3.6]_{\mathrm{ZO}-\mathrm{L}}=0.02 \pm 0.12 \mathrm{mag}$ and $[4.5]_{\mathrm{ZO}-\mathrm{L}}=0.12 \pm 0.07 \mathrm{mag}$, implying good agreement between the two sets of measurements.

\section{The search for $\sigma$ Orionis LT-type objects}

Field dwarfs with spectral types T0-8 (effective temperature $1400-700 \mathrm{~K})$ have typical colours of $I-J>4.5, J-H<1.5$, and $J-K_{\mathrm{s}}<2 \mathrm{mag}$ (Tinney et al. 2003; Zhang et al. 2009); the early types have redder $J-H$ and $J-K_{\mathrm{s}}$ colours and higher effective temperatures than the later types. By extrapolating the $\sigma$ Orionis cluster sequence using the field dwarf sequence, cluster members with a $\mathrm{T}$ spectral type appear to be at $J \sim 20$ mag (see Sect. 4 and Fig. 4). About the same apparent magnitude is found using the synthetic atmosphere $J$-band prediction of the 3-Myr COND model isochrone from Chabrier \& Baraffe (2000). However, when predicted bolometric luminosities and effective temperatures are transformed into the observable using relations for field dwarfs (see also Sect. 4 and Fig. 4), a $J$-band value of $\sim 21 \mathrm{mag}$ is found.

T-type objects of this magnitude will still be detected within the completeness of the ISAAC data, whereas they will be relatively faint or undetected in the less deep $I H K$-band images. For example, faint $\mathrm{T}$ type objects with $J=21.5, I-J>4.5$, $J-H<1$, and $J-K_{\mathrm{s}}<1 \mathrm{mag}$ will be undetected in all the optical images and only possibly detected in the near-infrared images of $\left(H\right.$ - or $K_{\mathrm{s}}$-band) limiting magnitudes fainter than $20.5 \mathrm{mag}$ $\left(\sim 470 \operatorname{arcmin}^{2}\right)$. Therefore, we opted for a search relying on the ISAAC $J$-band photometry, i.e., the deepest near-infrared photometry over the largest area, and with an automatic selection in terms of magnitudes and colours that is not too restrictive, to allow us to recover visually any potential cluster member candidate, including L-type objects.

First, we correlated the $\alpha-\delta$ coordinates of the $I J H K_{\mathrm{s}}-$ band sources using the IDL srcor procedure (IDL Astronomy User's Library, Landsman 1993); for each $J$-band source, we searched for the nearest counterpart within 2 arcsec in the $H$, $K_{\mathrm{s}}$, and $I$ bands. The correlations with the WFC- and LRIS $I$ band catalogues were performed separately. We then selected $19.5<J<21.5$ mag sources with no automatic $I$-band detection, or either $I>24 \mathrm{mag} \approx I_{\lim }$ (for unreliable or spurious

${ }^{2} \mathrm{~S}$ Ori 54 was not included in the comparison because in Luhman et al. (2008) the object was probably misidentified with the brighter source [SE2004] 26, at about 5 arcsec.

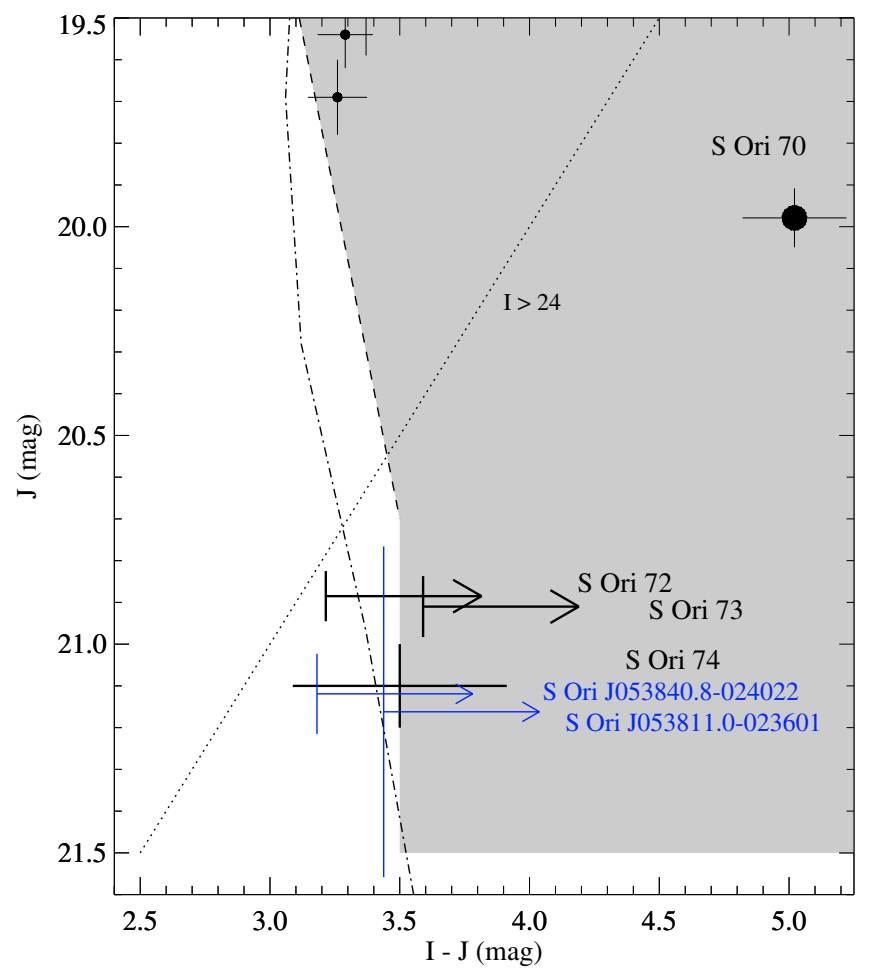

Fig. 3. $J$ versus $I-J$ colour-magnitude diagram. The shaded search region is where we expected cluster member candidates. The dashed line represents the extrapolated selection boundary from Caballero et al. (2007). The dotted line represents the $I>24$ mag selection sub-criterion. The dash-dot line represents the $3 \mathrm{Myr}$ COND model isochrone, where $I$ and $J$ are in the Cousins and CIT photometric systems, respectively. The two small filled circles are the faintest objects from Caballero et al. (2007) and the large filled circle is S Ori 70. The four $(5 \sigma)$ flux upper limits and one $(1 \sigma)$ error cross represent our new candidates. Probable galaxies are highlighted in blue.

detections) or $I-J>3.5 \mathrm{mag}$. As shown in Sect. 4, the $I-J$ colour is essential for distinguishing LT-type objects from galaxies. The $I-J>3.5 \mathrm{mag}$ sub-criterion intersects at $J=20.7 \mathrm{mag}$ with a linear extrapolation of the selection criterion applied by Caballero et al. (2007, see therein Fig. 2) for their sources with $I<I_{\mathrm{cmp}}=23 \mathrm{mag}$. For continuity between the searches, we also selected sources redder than their $I-J$ selection boundary and bluer than $3.5 \mathrm{mag}$. In the $J$ versus $I-J$ colour-magnitude diagram of Fig. 3, the shaded region represents the entire domain where we expected cluster member candidates, the dashed line represents the extrapolated selection boundary from Caballero et al. (2007), and the dotted line the $I>24$ mag sub-criterion. Finally, since the "2-j" Omega2000 J-band image (Table 2) overlaps with the northern ISAAC scans over $\sim 210 \mathrm{arcmin}^{2}$, we performed the selection process again for the sources with $J_{\text {ISAAC }}-J_{\text {Omega2000 }}>0.2$ mag and those without ISAAC counterparts.

About 800 sources were chosen by our selection criteria from our $I J$-band catalogues. We checked each source visually in all of the optical and infrared images, using the SAOImage DS9 display programme (Joye \& Mandel 2003) and commands in the X Public Access (XPA) messaging system ${ }^{3}$. The simultaneous visualisation in all available bandpasses and at all observing epochs allowed us to verify whether a source is real (or of low proper motion) and unresolved. Most sources were not detected automatically in the optical, because they are faint or very

\footnotetext{
${ }^{3}$ http://hea-www.harvard.edu/saord/xpa/
} 
close $^{4}$ to brighter ones, and their clearly bluer $I-J$ colours imply that they should be stars or unresolved galaxies. Many are spurious detections of spikes or glares in the $J$-band. Others represent charge persistencies of bright sources in the ISAAC detector (following precisely and chronologically the offsets of individual pointings), resolved galaxies, sources cut at image borders, or very blended sources, which are too close to bright stars in the optical to be identified. The "2-j" Omega2000 J-band sources with $J_{\text {ISAAC }}-J_{\text {Omega2000 }}>0.2$ mag were typically galaxies, resolved in the ISAAC images, whereas those without ISAAC counterparts were sources that could not be detected in the shallower survey region (see Sect. 2.2 and Fig. 2) and the gaps between the strips.

In a similar way, we searched for candidates in the additional areas of $\sim 15 \operatorname{arcmin}^{2}$ and $\sim 45 \operatorname{arcmin}^{2}$ represented by the shaded left and right regions in Fig. 2. These areas are common to the Omega2000 $J H_{\mathrm{s}_{\mathrm{s}}}$, WFC I-, and LRIS I-band data. The $J$-band data of the left and right regions (fields $2-\mathrm{j}$ and $18-\mathrm{j}$ ) are complete to 20.9 and $21.1 \mathrm{mag}$, respectively. They are therefore shallower by about 0.5 mag than the ISAAC data.

These searches allowed us to find four sources that are indeed undetectable by eye in the deepest $I$-band images (see Sect. 4), and a half-dozen of sources at $J>20.7 \mathrm{mag}$ that are barely detected beyond the $I$-band limiting magnitudes. Most of the latter sources appear to be bluer than $I-J \sim 3.5$ mag. They have magnitude errors $\$ 0.1 \mathrm{mag}$ in the $J H K_{\mathrm{s}}$-bands and red colours of $J-H \approx 1 \mathrm{mag}$ or $J-K_{\mathrm{s}} \approx 2 \mathrm{mag}$. Only one of them has a colour $I-J \geq 3.5 \mathrm{mag}$. It was selected as a candidate (see Sect. 4), whereas the others were rejected because they are probable galaxies or faint field M- or early L-type dwarfs. Some sources could not be verified in the $I$-band images because of blending with extended stellar spikes and glares. We estimated that areas of $\sim 10$ and $\sim 5 \operatorname{arcmin}^{2}$ are lost in the main- and additional areas, respectively. Thus, the total search area with $J$-band completeness $\geq 21.1 \mathrm{mag}$ (ISAAC and Omega2000 18-j data) amounts to $\sim 790 \operatorname{arcmin}^{2}$.

\section{Results and discussion}

Besides recovering the two faintest cluster member candidates ${ }^{5}$ S Ori J053932.4-025220 and S Ori J054011.6-025135 from Caballero et al. (2007) and the T-type S Ori 70, we detect three new L- and T-type candidates and two probable galaxies (see finding charts of Figs. B.1-B.4), among many other objects rejected because they do not meet our selection criteria. As shown in Fig. 3, the new candidates are about one magnitude fainter than S Ori 70. The photometric information that we compiled from the images of different depths are listed in Tables 4 and 5, where the $5 \sigma$ flux upper limits correspond to the magnitude limits of the images.

In the $J$ versus $J-[3.6]$ and $J-[4.5]$ colour-magnitude diagrams of Figs. 4 and 5, we represent the candidates together with known $\sigma$ Orionis cluster members and candidates (Caballero et al. 2007; Zapatero Osorio et al. 2007, 2008). The solid line represents the spectrophotometric sequence of field mid-M- to late-T-type dwarfs, shifted to match the brightness of

\footnotetext{
${ }^{4}$ De-blended in the images subtracted by the PSF fitted sources (NOAO.DIGIPHOT .DAOPHOT . ALLSTAR) or subtracted by a smoothing obtained with a moving average box much smaller than the image size (NOAO. IMRED . CCDRED. MKSKYCOR).

${ }^{5}$ For the latter, we were able to measure $H=18.8 \pm 0.2 \mathrm{mag}$ and $K_{\mathrm{s}}=18.5 \pm 0.1 \mathrm{mag}$, implying early L colours $J-H=0.8 \mathrm{mag}$ and $J-K_{\mathrm{s}}=1.1 \mathrm{mag}$.
}

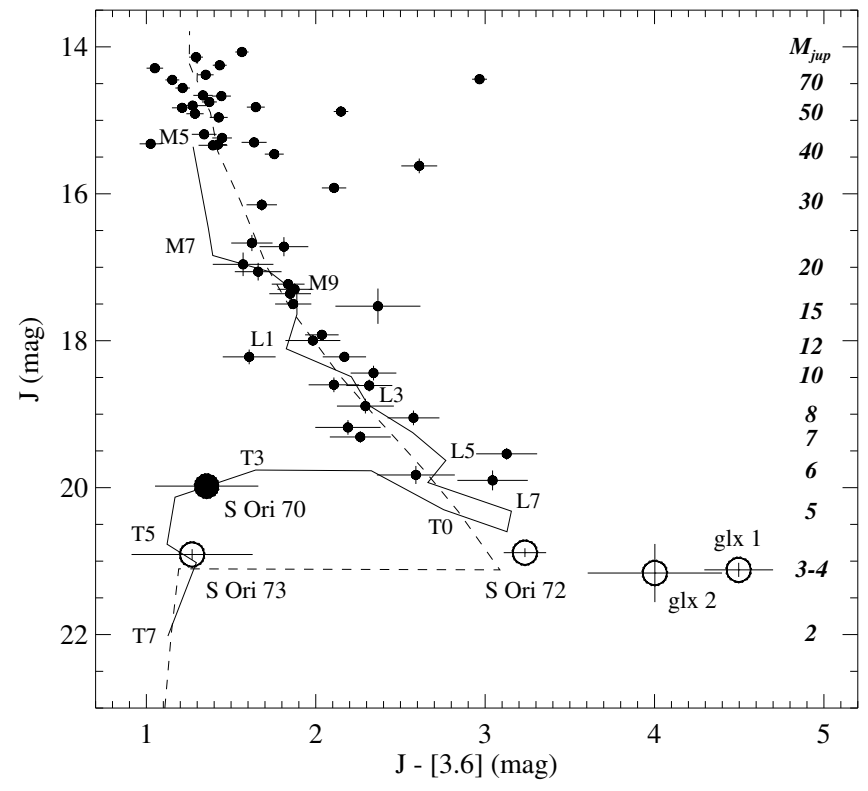

Fig. 4. $J$ versus $J-[3.6]$ colour-magnitude diagram of $\sigma$ Orionis cluster members and candidates. The filled circles correspond to objects from Caballero et al. (2007) and Zapatero Osorio et al. (2007, 2008). The large filled circle is S Ori 70. The large open circles are the new candidates. S Ori J053840.8-024022 and S Ori J053811.0-023601 are labelled "glx 1" and "glx 2", respectively. The solid line represents the field dwarf sequence shifted to match the brightness of the late-Mtype $\sigma$ Orionis cluster members. The dashed line represents the $3 \mathrm{Myr}$ COND model isochrone (see text); masses are indicated to the right in units of Jupiter masses.

the late-M-type cluster members (Zapatero Osorio et al. 2008). For the field dwarfs, we use average absolute $I$-band magnitudes, $I-J$, and $J-K_{\mathrm{s}}$ colours compiled by Caballero et al. (2008a) ${ }^{6}$, $J-H$ colours from Vrba et al. (2004) ${ }^{7}$, and mid-infrared magnitudes from Patten et al. (2006). In Fig. 4, the dashed line represents the 3 Myr COND model isochrone at the cluster distance, adapted by converting predicted effective temperature and luminosity into observables using relations for field dwarfs (procedure explained in Zapatero Osorio et al. 2008).

We also represent the candidates in $I-J$ versus $J-H$ and $J-K_{\mathrm{s}}$ colour-colour diagrams (Figs. 6 and 7) as well as in various diagrams with mid-infrared filters (Figs. 8-10), together with sources from the GOODS-MUSIC catalogue (Grazian et al. 2006) that we use as a control field to study the potential contamination by extragalactic sources in our survey. In all the figures, the open circles represent the new candidates (labelled) and the solid line represents part of the field LT-type dwarf sequence. The GOODS-MUSIC survey is centred on

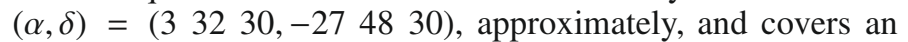
area of $143.2 \mathrm{arcmin}^{2}$ (except in the $H$-band, where the area is $78 \operatorname{arcmin}^{2}$ ), i.e., less than a fifth of our search area. The limiting magnitudes are $i=26.1, J=23.6, H=22.9, K_{\mathrm{s}}=21.9$, $[3.6]=21.2,[4.5]=20.1,[5.8]=18.3$, and $[8.0]=17.6 \mathrm{mag}$,

6 Note that the photometric values in Table 3 therein correspond
to the spectral types $\mathrm{M} 3 \mathrm{~V}, \mathrm{M} 4 \mathrm{~V}, \mathrm{M} 5 \mathrm{~V}, \ldots$ instead of $\mathrm{M} 3-4 \mathrm{~V}, \mathrm{M} 4-5 \mathrm{~V}$,
$\mathrm{M} 5-6 \mathrm{~V}, \ldots$ The $I$ and $J K_{\mathrm{s}}$ magnitudes are in the Johnson-Cousins- and
$2 \mathrm{MASS}$ photometric systems, respectively.
7 The $J-H$ colour is transformed back from the CIT- to the $2 \mathrm{MASS}$
photometric system using the same colour transformation of Carpenter (2001) as used in Vrba et al. (2004). 
Table 4. Coordinates and photometry of the new L- and T-type cluster member candidates ${ }^{a}$.

\begin{tabular}{|c|c|c|c|c|c|c|c|c|c|c|}
\hline Name & $\begin{array}{c}\alpha \\
(\mathrm{J} 2000)\end{array}$ & $\begin{array}{c}\delta \\
(\mathrm{J} 2000)\end{array}$ & $\begin{array}{c}I \\
(\mathrm{mag})\end{array}$ & $\begin{array}{c}Z \\
(\mathrm{mag})\end{array}$ & $\begin{array}{c}J \\
(\mathrm{mag})\end{array}$ & $\begin{array}{c}H \\
(\mathrm{mag})\end{array}$ & $\begin{array}{c}K_{\mathrm{s}} \\
(\mathrm{mag})\end{array}$ & $\begin{array}{c}{[3.6]} \\
\text { (mag) }\end{array}$ & $\begin{array}{c}{[4.5]} \\
\text { (mag) }\end{array}$ & $\begin{array}{c}{[5.8]} \\
\text { (mag) }\end{array}$ \\
\hline S Ori 72 & 53859.17 & -23526.0 & $>24.1$ & $>23.3$ & $20.89 \pm 0.06$ & $19.57 \pm 0.03$ & $18.72 \pm 0.05$ & $17.65 \pm 0.11$ & $17.51 \pm 0.29$ & $17.28 \pm 0.38$ \\
\hline S Ori 73 & 53814.49 & -24511.8 & $>24.5$ & $23.5 \pm 0.5$ & $20.91 \pm 0.07$ & $20.83 \pm 0.12$ & $20.91 \pm 0.15$ & $19.64 \pm 0.35$ & $18.77 \pm 0.35$ & $>16.0$ \\
\hline S Ori 74 & 53844.27 & -24007.9 & $24.6 \pm 0.4$ & $>23.3$ & $21.1 \pm 0.1$ & $>18.54^{b}$ & $19.38 \pm 0.10$ & $\ldots{ }^{c}$ & $\ldots^{c}$ & $>16.0$ \\
\hline
\end{tabular}

${ }^{a}$ All have [8.0] > 15.1 mag. ${ }^{b}$ Lower magnitude limit computed for the UKIDSS GCS specific field. ${ }^{c}$ Blended with a spike from the star Mayrit 260182 (located at 11.8 arcsec).

Table 5. Coordinates and photometry of probable galaxy candidates ${ }^{a}$.

\begin{tabular}{cccccccccc}
\hline \hline Name & $\begin{array}{c}I \\
(\mathrm{mag})\end{array}$ & $\begin{array}{c}Z \\
(\mathrm{mag})\end{array}$ & $\begin{array}{c}J \\
(\mathrm{mag})\end{array}$ & $\begin{array}{c}H \\
(\mathrm{mag})\end{array}$ & $\begin{array}{c}K_{\mathrm{s}} \\
(\mathrm{mag})\end{array}$ & $\begin{array}{c}{[3.6]} \\
(\mathrm{mag})\end{array}$ & $\begin{array}{c}{[4.5]} \\
(\mathrm{mag})\end{array}$ & $\begin{array}{c}{[5.8]} \\
(\mathrm{mag})\end{array}$ & $\begin{array}{c}{[8.0]} \\
(\mathrm{mag})\end{array}$ \\
\hline S Ori J053840.8-024022 & $>24.3$ & $\ldots$ & $21.12 \pm 0.10$ & $19.92 \pm 0.07$ & $18.94 \pm 0.08$ & $16.62 \pm 0.18$ & $16.29 \pm 0.19$ & $>16.0$ & $>15.1$ \\
S Ori J053811.0-023601 & $>24.6$ & $>22.9$ & $21.16 \pm 0.40$ & $20.66 \pm 0.11$ & $19.61 \pm 0.09$ & $17.16 \pm 0.02$ & $16.53 \pm 0.04$ & $15.56 \pm 0.06$ & $14.69 \pm 0.12$ \\
\hline
\end{tabular}

${ }^{a} \mathrm{~S}$ Ori J053811.0-023601 is at 28.0 arcsec from S Ori 70.

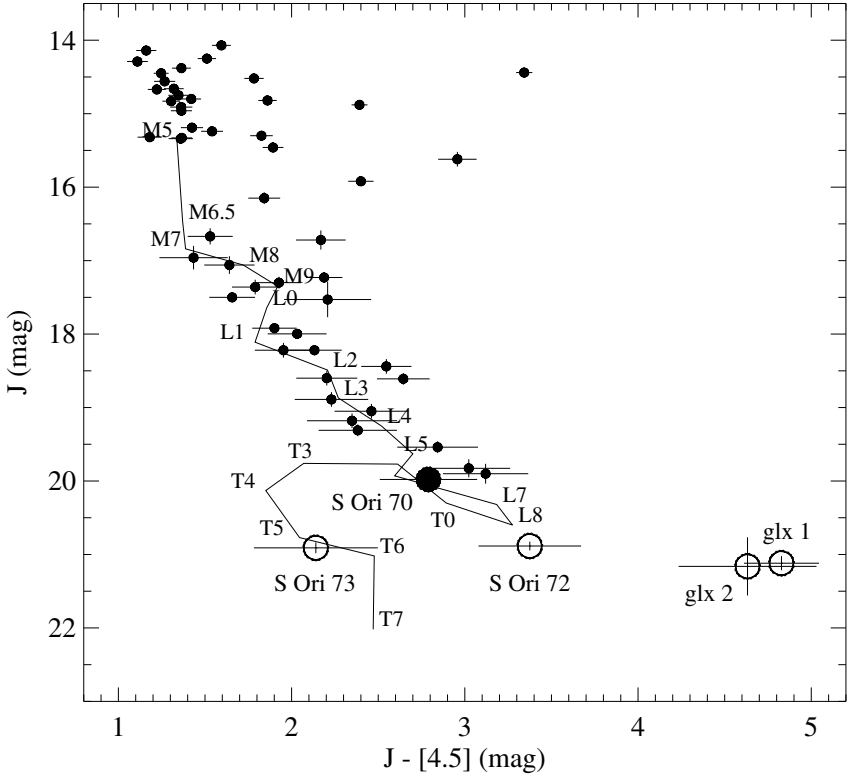

Fig. 5. $J$ versus $J-[4.5]$ colour-magnitude diagram. Same as in Fig. 4.

when converted from the AB system to the Vega system ${ }^{8}$. In the magnitude range $19.5<J<21.5 \mathrm{mag}$, the catalogue contains 882 galaxies, 37 active galactic nuclei (AGN), and 83 stars, and in the smaller $H$-band area, 505 galaxies, 27 AGN, and 49 stars. Stars and AGN are distinguished from "normal" galaxies mostly by morphological and photometric criteria, or else by spectroscopic criteria. Stars are distinguished from AGN by spectroscopic criteria. In the infrared colour-colour diagrams of Figs. 9

\footnotetext{
8 The $i$ - (or $F 775 W$ ) band photometry is from HST/ACS and similar to that from the Sloan Digital Sky Survey (SDSS). A transformation $I_{\text {Cousins }}=i_{\text {SDSS }}-0.3780 *\left(i_{\text {SDSS }}-z_{\text {SDSS }}\right)-0.3974(\sigma=0.0063 \mathrm{mag})$ has been obtained for stars (http://web.archive.org/web/ 20071014232413/http://www.sdss.org/dr6/algorithms/ sdssUBVRITransform.html); we assumed that this transformation is also valid for galaxies. The VLT/ISAAC $J H K_{\mathrm{s}}$-band photometry was converted to the Vega system using the transformations provided in the web page http://web.archive.org/web/20070814141037/ http://www . eso.org/science/goods/releases/20050930/; it is found to be consistent within 0.05 mag with the 2MASS point-source photometry. For the Spitzer/IRAC [3.6][4.5][5.8][8.0]-band photometry, we used the transformations provided in the web page http:// web.ipac. caltech. edu/staff/gillian/cal.html
}

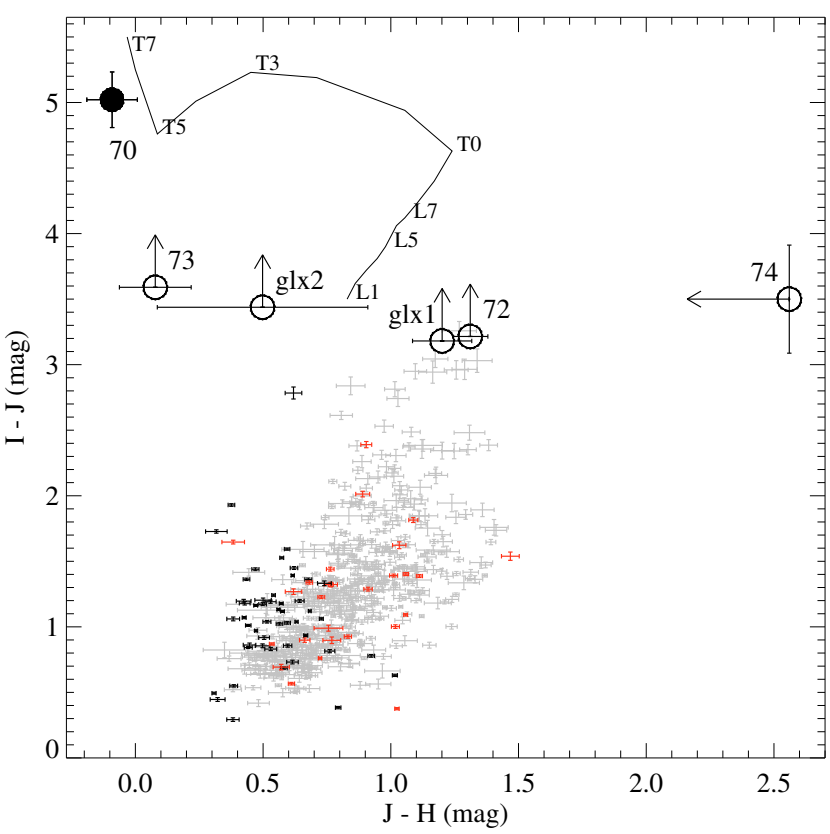

Fig. 6. $I-J$ versus $J-H$ colour-colour diagram of stars (black crosses), AGN (red crosses), and galaxies (grey crosses) from the GOODS-MUSIC catalogue (Grazian et al. 2006), in the magnitude range $19.5<J<21.5 \mathrm{mag}$. The solid line represents the field L1-T7type dwarf sequence, the filled circle represents S Ori 70, and the open circles represent the new candidates. S Ori J053840.8-024022 and S Ori J053811.0-023601 are labelled "glx 1" and "glx 2", respectively.

and 10, the sequence of mid-L- to early-T type field dwarfs overlaps with the domain of galaxies and AGN; only T type dwarfs tend to have different colours. The colour-magnitude diagrams of Fig. 8 also indicate that, from $J=19.5$ to $21.5 \mathrm{mag}$, the colour ranges of galaxies and AGN become broader and the number of galaxies increases (here by a factor of 1.4 in a 0.5 -mag interval). However the optical-infrared diagrams of Figs. 6 and 7 show that mid-L to mid-T type dwarfs are clearly redder in $I-J$ than the other sources. Thus, the $I-J$ colour is essential to distinguishing these objects from galaxies and AGN, whereas the infrared colours only help us to guess the spectral type. 


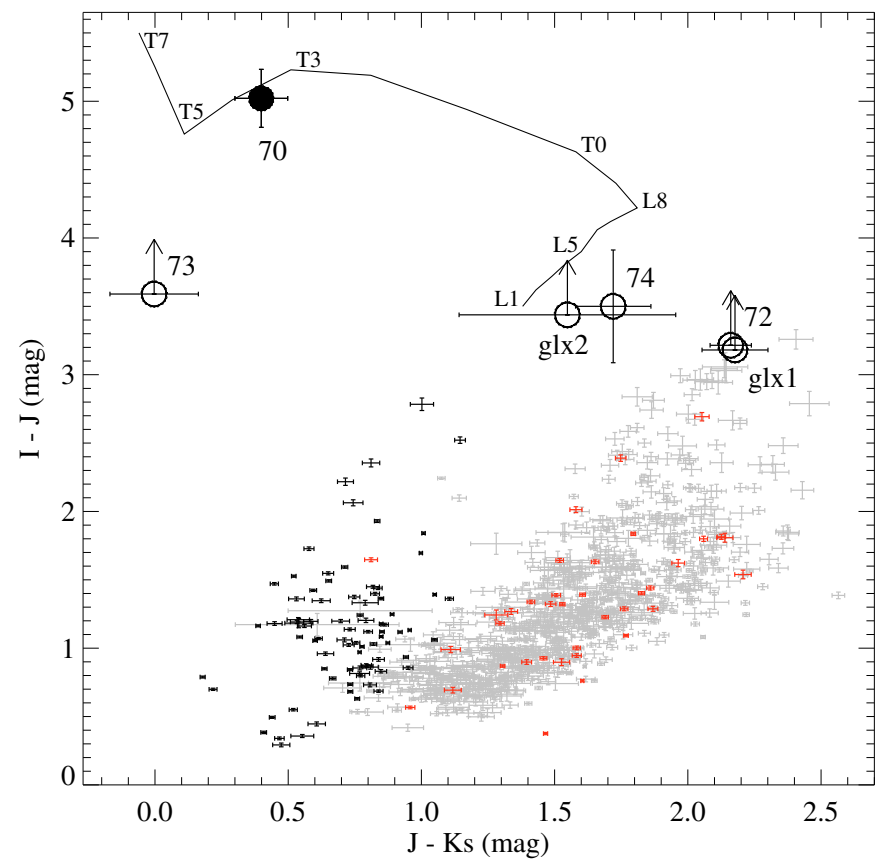

Fig. 7. $I-J$ versus $J-K_{\mathrm{s}}$ colour-colour diagram. Same as in Fig. 6 .

\subsection{New L- and T-type candidates}

S Ori 72 , with $J-H \approx 1.3 \mathrm{mag}$ and $J-K_{\mathrm{s}} \approx 2.2 \mathrm{mag}$, could be a late L-type object. It is clearly detected in the ISAAC $J$-band image from December 2001 and even better in Omega2000 $\mathrm{HK}_{\mathrm{s}}$ band images (observations 2-h and 2-k, Table 2), whereas it is slightly blended but detected in the [3.6][4.5][5.8]-band images (its visual neighbour is 2MASS J05385930-0235282, a field star located 3 arcsec south-east, with $J \approx 16.0 \mathrm{mag}$ and $I-J \approx 0.9 \mathrm{mag}$ ). S Ori 72 is undetected in the WFC $I$-band image and the LRIS IZ-band images (1998iz3, Table 1). The $F W H M \mathrm{~s}$ in the $J H K_{\mathrm{s}}$-band images are approximately equal to those of nearby faint point-like sources, of about $0.5,1.2$, and 0.8 arcsec, respectively. Among the new candidates presented in this paper, S Ori 72 is the only one that is detected in the GCSUKIDSS images, with $K_{\text {UKIDSS }}=18.60 \pm 0.28 \mathrm{mag}$, in agreement with our measurement. In Fig. 11, we show its spectral energy distribution, together with average ones of field dwarfs of L7- and L8 spectral types (dotted lines). S Ori 72 is relatively brighter in the $H K_{\mathrm{s}}$-bands. A preliminary measurement of S Ori 72's proper motion using the ISAAC-Omega2000 images of $3.87 \mathrm{yr}$ time baseline and the method described in Bihain et al. (2006) allows us to impose a $2 \sigma$ upper limit of 30 mas/yr. Although the estimate should be improved before comparison with the $\mu \lesssim 5-10$ mas $\mathrm{yr}^{-1}$ amplitude of $\sigma$ Orionis members (Caballero 2007), it indicates that S Ori 72 is not a high-proper motion object and unlikely to be a nearby $(\leq 30 \mathrm{pc})$ source. From the $J$ versus $J-[3.6]$ and $J-[4.5]$ colour-magnitude diagrams of Figs. 4 and 5, S Ori 72 could be an L/T transition cluster member candidate, but Figs. 6-10 imply that it could also be a galaxy or an AGN.

With $J-H \sim J-K_{\mathrm{s}} \sim 0$ mag, SOri 73 has near-infrared colours of a mid T-type object. It is clearly detected in the ISAAC $J$-band image, but appears very faint in the WFC Z-band image from November 2008, the CFHTIR $H K^{\prime}$-band images from February 2004 (20-h and 20-k, Table 3), and the public Spitzer/IRAC [3.6]- and [4.5]-band images. It is undetected in the WFC I-band image, the LRIS IZ-band images (2000i2, 1998iz2, Table 1), and in the Omega2000 $H K_{\mathrm{s}}$-band images (17-h and 17-k). Its $F W H M$ in the $J$-band image is approximately equal to that of nearby faint point-like sources, of about 0.5 arcsec. In Fig. 11, we show its spectral energy distribution, together with average ones of field dwarfs of T4 and T6 spectral types. The position of S Ori 73 in the $J$ versus $J-[3.6]$ diagram of Fig. 4 agrees with both adapted field- and model sequences, securing this source as a good T-type- and cluster member candidate. The colour-colour diagrams of Figs. 6, 7, and 9 also indicate that there are neither stars, nor AGN, nor galaxies in GOODS-MUSIC as red in $I-J$ and blue in $J-H$ and $J-K_{\mathrm{s}}$ as this object.

With $I-J=3.5 \pm 0.4 \mathrm{mag}$ and $J-K_{\mathrm{s}} \approx 1.7 \mathrm{mag}$, S Ori 74 could be an L-type object. It is detected in the ISAAC $J$-band image and in an Omega2000 $K_{\mathrm{s}}$-band image (18-k, Table 3$)$. The object appears point-like in both images with a typical local FWHM of 0.7 and 0.9 arcsec, respectively, whereas it appears faint and blended with a stellar spike in the [3.6][4.5][5.8]band images, preventing us from determining its IRAC photometry. The candidate is barely detected in the LRIS I-band images (2000i3 and 1998i3, Table 1) and undetected in a stellar glare in the WFC I-band- and LRIS Z-band images (1998z3). In Fig. 11, we show its spectral energy distribution, together with average ones of field dwarfs of L2- and L8 spectral types. From Fig. 7, it could also be a galaxy or an AGN. However, S Ori 74 is located 11.8 arcsec north ( 4250 AU; open square in Fig. 2) of the bright K7.5-type cluster star Mayrit 260182, 4.3 arcmin south of the cluster centre. The probability of chance alignment is only $5 \%$ for cluster members at this angular distance from the $\sigma$ Orionis centre (Caballero 2009, Fig. 1). Mayrit 260182, Mayrit 270181, and Mayrit 277181 also form a possible triple system (Caballero 2006). Caballero et al. (2006) previously proposed that S Ori $68+$ SE 70 is a planet-brown dwarf system candidate, of greater mass ratio and smaller separation. There are, however, known red galaxies at comparable angular separations to $\sigma$ Orionis cluster members. For example, the type I obscured quasi-stellar object UCM 0536-0239 is located about 14.9 arcsec south of the T Tauri star Mayrit 97212 (Caballero et al. 2008b). Observational follow-up is necessary to confirm whether S Ori 74 is a cluster member and companion of Mayrit 260182.

\subsection{Probable galaxy candidates}

With $J-H \approx 1.2 \mathrm{mag}$ and $J-K_{\mathrm{s}} \approx 2.2 \mathrm{mag}$, SOri J053840.8-024022 could be a late L-type object or a galaxy. It is detected with brighter magnitudes at longer wavelengths, from the Omega2000 $\mathrm{JHK}_{\mathrm{s}}$-band images $(18 \mathrm{j}-\mathrm{k}$, Table 3) to the [3.6][4.5]-band images, but is then undetected in the [5.8][8.0]-band images. It is undetected in the WFC and LRIS I-band images (2000i3, Table 1). In Fig. 11, we show its spectral energy distribution, together with average ones of field dwarfs of L7- and L8 spectral types. In the ISAAC J-band image, it is found to be slightly extended and fainter than in the lower-resolution Omega2000 J-band image. The FWHMs of the object in the Omega2000 JHK $K_{\mathrm{s}}$-band images are systematically larger by a factor $\approx 1.4$ than those of nearby faint point-like sources, suggesting that it is a galaxy. S Ori J053840.8-024022 could be an L/T transition object, but from the $J$ versus $J-[3.6]$ and $J-[4.5]$ diagrams (Figs. 4 and 5), it is redder than the expected sequence of the cluster. Galaxies from the GOODSMUSIC catalogue with these red colours appear at magnitudes $J \gtrsim 20.5$ mag (Fig. 8). Figure 9 illustrates its infrared excess in the $K_{\mathrm{s}^{-}}$, [3.6]-, and [4.5]-bands relative to the field dwarf sequence, and also suggests that this object is likely to be a galaxy. 

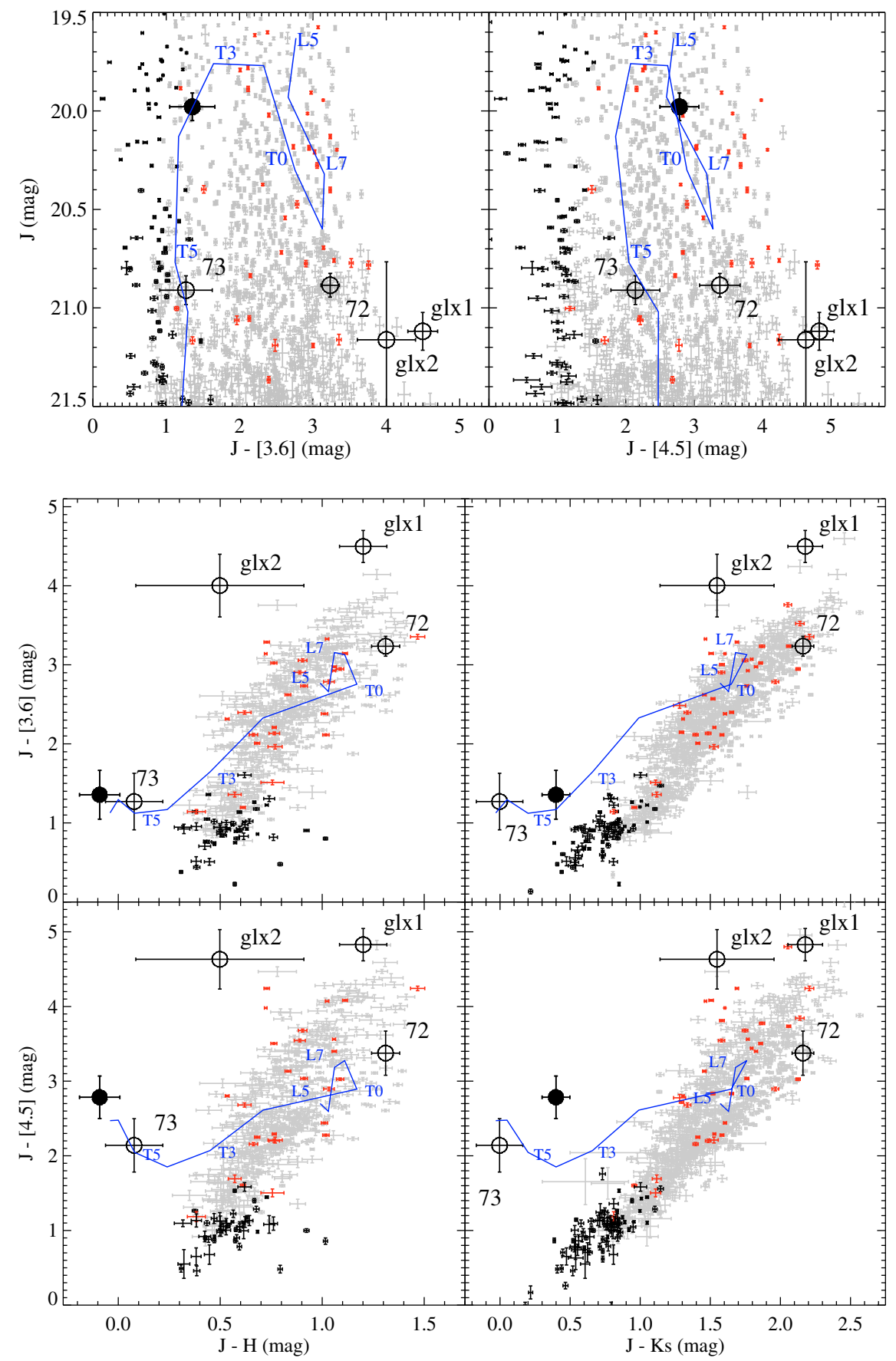

Fig. 8. $J$ versus $J-[3.6]$ and $J-[4.5]$ colour-magnitude diagrams. Same as in Fig. 6. The blue solid line represents the field dwarf sequence shifted as in Fig. 4.
Fig. 9. Near- and mid-infrared colour-colour diagrams: $J-[3.6]$ and $J-$ [4.5] versus $J-H$ (topand bottom left), $J-[3.6]$ and $J-[4.5]$ versus $J-K_{\mathrm{s}}$ (top- and bottom right). Same as in Fig. 6.
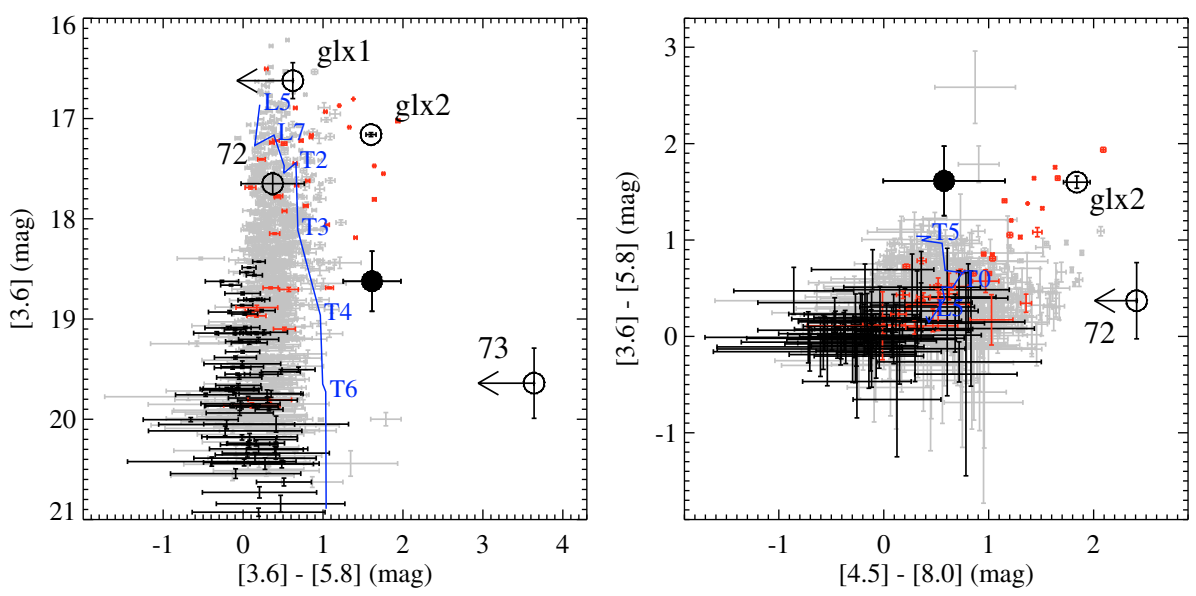

Fig. 10. Mid-infrared diagrams: [3.6] versus [3.6]-[5.8] (left), [3.6]-[5.8] versus $[4.5]-[8.0]($ right). Same as in Fig. 6. 


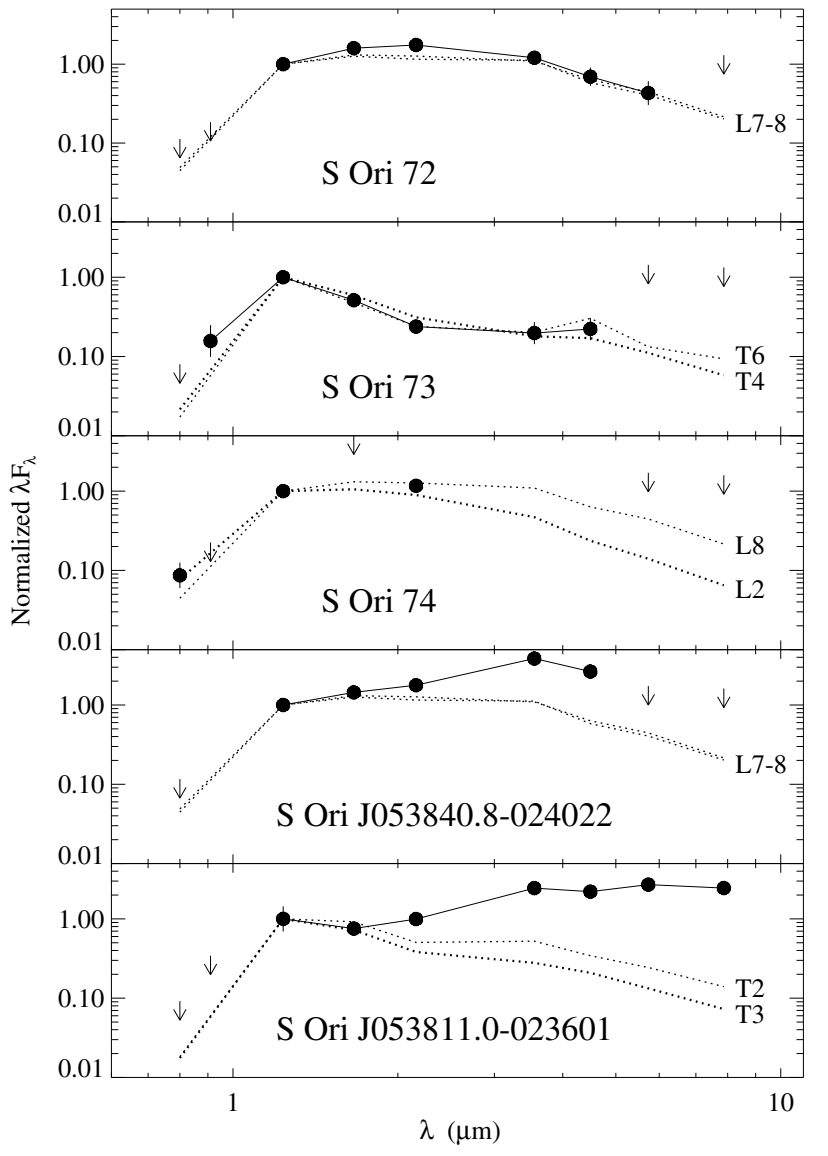

Fig. 11. Spectral energy distributions of the new candidates compared to average ones of field dwarfs (dotted lines). $\operatorname{IZJHK}_{\mathrm{s}}[3.6][4.5][5.6][8.0]$ from left to right.

SOri J053811.0-023601, with $J-H \sim 0.5 \mathrm{mag}$ and $J-K_{\mathrm{s}} \sim 1.5 \mathrm{mag}$, could be an early T-type object or a galaxy. It is detected with brighter magnitudes at longer wavelengths, from the Omega2000 $J H K_{\mathrm{s}}$-band images $(18 \mathrm{j}-\mathrm{k}$, Table 3$)$ to the [3.6][4.5][5.6][8.0]-band images. It is undetected in the WFC Iband image and the LRIS IZ-band images (2000i1 and 1998iz1, Table 1). In Fig. 11, we show its spectral energy distribution, together with average ones of field dwarfs of T2 and T3 spectral types. We caution that the object appears relatively faint in the $J$-band and that the $H K_{\mathrm{s}}$-band centroids are 0.8 arcsec south of the $J$-band centroid, although the $J H$-band data were obtained on the same observing night. The FWHMs in the $H K_{\mathrm{s}}-$ band images are approximately equal to those of nearby faint point-like sources, of about 1.2 and 0.9 arcsec, respectively. S Ori J053811.0-023601 appears as an L/T transition object, but from the $J$ versus $J-[3.6]$ and $J-[4.5]$ diagrams (Figs. 4 and 5) and as for S Ori J053840.8-024022, it is redder than the expected cluster sequence and could be a galaxy (Fig. 8). S Ori J053811.0-023601 is particularly bright in the [5.8]- and [8.0]-bands and displays a colour [3.6]-[8.0] $=2.5 \pm 0.1$, redder than most $\sigma$ Orionis low-mass member candidates (Zapatero Osorio et al. 2007; Scholz \& Jayawardhana 2008; Luhman et al. 2008). Considering that $\geq 50 \%$ of the known $\sigma$ Orionis planetary-mass candidates exhibit excesses longward of $5 \mu \mathrm{m}$ (Zapatero Osorio et al. 2007), it appears to be a cluster member. In Figs. 6, 7, and 9, its optical and near-infrared colours differ from those of AGN and galaxies, but in Fig. 10, its other colours are consistent with the AGN hypothesis (see also Fig. 1 in Stern et al. 2005, representing spectroscopically identified stars, AGN, and galaxies). Hence, although we cannot exclude completely this source beeing a peculiar cluster member with extreme infrared excesses, our data seem to indicate that it is more probably an AGN.

\subsection{Cluster membership}

Because our search area is larger than that of the GOODSMUSIC catalogue, contamination by red galaxies and AGN is even more likely to explain some of our candidates. Caballero et al. (2008b) present low-resolution optical spectroscopy and spectral energy distributions between 0.55 and $24 \mu \mathrm{m}$ of two sources fainter than the star-brown-dwarf cluster boundary, which were interpreted to be peculiar $\sigma$ Orionis members with very red colours related to discs. They are instead two emissionline galaxies at moderate redshift, one with an AGN and the other ongoing star formation. In the present study, we assume that S Ori J053840.8-024022 and S Ori J053811.0-023601 are galaxy- or AGN contaminants and that the other objects are Galactic candidates awaiting confirmation by higher resolution imaging, proper motion, or spectroscopy.

In our search, we must also account for contamination by field dwarfs. Caballero et al. (2008a) provide predictions of the number of L5-T0, T0-T5, and T5-T8 field dwarf contaminants per square degree towards the $\sigma$ Orionis region, in onemagnitude $I$-band intervals and from $I=21.0$ to $29.0 \mathrm{mag}$. We convert the bright and faint boundaries of the range $J=$ 19.7-21.1 mag (as a prolongation of the search range of Caballero et al. 2007) into the I-band magnitudes corresponding to the earliest and latest spectral types of each of the three contaminant groups. We then sum the predicted numbers of contaminants accounting for the $I$-band ranges and scale the sums to the search area that is complete to $J \geq 21.1 \mathrm{mag}\left(\sim 790 \mathrm{arcmin}^{2}\right)$. We obtain about three L5-T8-type field dwarfs, which all contribute the most to the light close to $J=21.1 \mathrm{mag}$. This predicted value remains mostly indicative, because the initial mass function and scale heights of late L- and T-type dwarfs are still uncertain. Interestingly, Caballero et al. (2008a) assume a rising mass function in the planetary mass regime and predict spatial densities of T0-8 dwarfs that are a factor of two higher than those derived from observations (Metchev et al. 2008; Lodieu et al. 2009a).

S Ori 73 and S Ori 70 are located ${ }^{9}$ at 11.9 and 8.7 arcmin from $\sigma$ Ori $\mathrm{AB}$, respectively. S Ori 72 and S Ori 74 are closer, at 3.6 and 4.1 arcmin, respectively. Interestingly, the location of these faintest, presumably least massive candidates contrasts with that of the eleven 13-6 $M_{\text {Jup }}$ free-floating planetary-mass candidates from Caballero et al. (2007), further out at 26-13 arcmin in the survey area (see Fig. 2). Caballero (2008a) find an apparent deficit of low mass objects $\left(M<0.16 M_{\odot}\right)$ towards the $\sigma$ Orionis cluster centre. If the cluster membership census and the individual masses are confirmed, this configuration could be explained by several mechanisms, including e.g., a possible photo-erosion by the central OB stars (Hester et al. 1996; Whitworth \& Zinnecker 2004) in the deep gravity well. Complementary studies of the dense cluster core (Bouy et al. 2009) and other cluster regions could thus help us to understand the formation of low-mass planetary-mass objects.

\footnotetext{
9 S Ori 73 is the only candidate found in one of the deeper multi-band search areas, summing up to $\sim 470 \operatorname{arcmin}^{2}$, see Sect. 3 .
} 


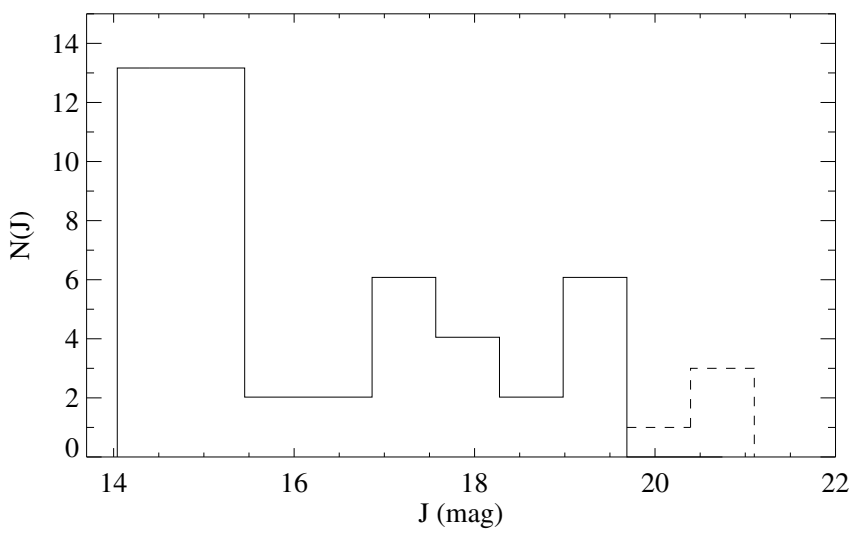

Fig. 12. $J$-band luminosity function with the LT-type candidates at $J>$ 19.7 mag (dashed line) and the brighter cluster member candidates from Caballero et al. (2007) scaled to the search area (solid line).

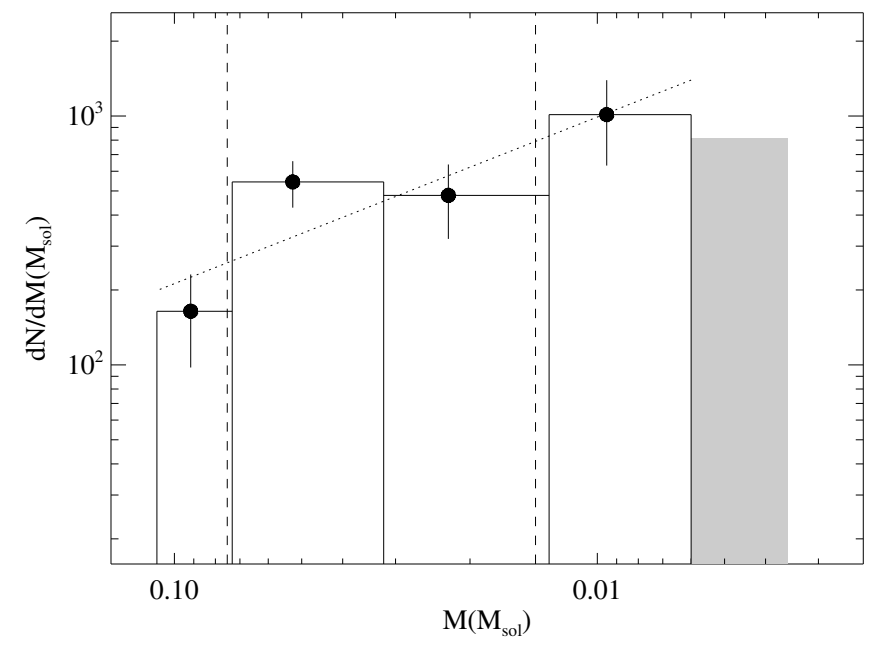

Fig. 13. Mass spectrum with contamination-corrected data. The dotted segment represents the linear fit to the data points from Caballero et al. (2007) in the mass range $0.11-0.006 M_{\odot}$, which are previously scaled to the search area. The shaded region is our estimate of 0-2 cluster members in the mass range $0.006-0.004 M_{\odot}$. From left to right, the vertical dashed lines represent the hydrogen and deuterium burning mass limits, respectively.

\subsection{Mass spectrum}

We consider the luminosity and mass functions for the ISAACand additional areas, where the search is complete down to $J \geq$ $21.1 \mathrm{mag}\left(\sim 790 \operatorname{arcmin}^{2}\right)$.

In Fig. 12, we show the $J$-band luminosity function. The magnitude bins in the range $J=19.7-21.1$ mag correspond to the three new LT-type candidates and S Ori 70 (dashed line). The magnitude bins in the range $J=14.1-19.7$ mag correspond to the cluster member candidates from Caballero et al. (2007), i.e., in the ISAAC area; they are scaled by the area factor $(790) / 780=1.0128$. The magnitude bins have equal widths of about $0.7 \mathrm{mag}$.

We estimate the masses of our new cluster member candidates by comparing with the theoretical bolometric luminosities from the Lyon group (e.g., Baraffe et al. 2003), using exactly the same method as in Caballero et al. (2007). If cluster members, S Ori 72-74 would each have an estimated theoretical mass of $4_{-2}^{+3} M_{\text {Jup }}$, accounting for age, distance, and photometric uncertainties. This rounded up result does not change significantly by using a cluster distance of 400 pc (Mayne \& Naylor 2008) or 440 pc (Sherry et al. 2008) instead of 360 pc (Brown et al. 1994). The effective temperature corresponding to that mass would be of $\sim 1400 \mathrm{~K}$. In Fig. 13, we display the mass spectrum $(\Delta N / \Delta M)$. The filled circles represent the contaminationcorrected data points from Caballero et al. (2007) scaled to the search area of $\sim 790 \mathrm{arcmin}^{2}$. The last bin (shaded region) corresponds to the result from the present study for the magnitude range $J=19.7-21.1 \mathrm{mag}$. Subtracting the three possible contaminants (see Sect. 4.3) from the four LT-type candidates and accounting for the Poissonian error, we estimate $0-2$ cluster members with a mass of $0.006-0.004 M_{\odot}$.

Previous studies of the substellar population in the $\sigma$ Orionis cluster find that the mass spectrum increases toward lower masses. Béjar et al. (2001) show that it can be represented by a potential law $\left(\Delta N / \Delta M \propto M^{-\alpha}\right)$ with an $\alpha$ index of 0.8 in the mass range $0.11-0.013 M_{\odot}$. González-García et al. (2006) and Caballero et al. (2007) extend this mass spectrum to $0.006 M_{\odot}$ and find a slightly lower index $\alpha=0.6$. For the substellar mass range of 0.073-0.006 $M_{\odot}$, Caballero et al. (2007) obtain an even lower $\alpha$ index of 0.4 . An extrapolation of the mass spectrum with an index $\alpha=0.4-0.8$ predicts $3-7$ objects in the mass range $0.006-0.004 M_{\odot}$. From our survey, the most likely number of cluster members in this mass interval is in the range $0-2$. This could be an indication of a turnover in the substellar mass spectrum. However, given the low statistics and the possibility that the number of contaminants could be overestimated, such a change in the slope of the mass spectrum should be considered with caution. If real, the turnover could be related to an opacity mass limit, turbulence effects, or a different mass-luminosity relation (if less massive objects were fainter than predicted). Wider and deeper searches would be very valuable in constraining the mass spectrum more reliably at these and lower masses.

\section{Conclusions}

The mass function in young open clusters can provide clues about the formation mechanism of free-floating planetarymass objects. We therefore decided to explore the substellar mass function for $M<6 M_{\text {Jup }}$ in the $\sim 3$ Myr old $\sigma$ Orionis open cluster. We extended to $J=19.5-21.5 \mathrm{mag}$ the $\sim 780 \mathrm{arcmin}^{2}$ INT/WFC-VLT/ISAAC $I J$-band search of Caballero et al. (2007). $J$-band sources (ISAAC and CAHA $3.5 \mathrm{~m} /$ Omega2000) were cross-matched with $I$ - (WFC and Keck/LRIS) and $H K$-band sources (Omega2000, NTT/SofI, WHT/LIRIS, and CFHT/CFHTIR). We selected sources redder than a boundary at $I-J>3.1-3.5$ or without an $I$-band detection or fainter than $I=24$ mag. These sources were then checked visually in all available images, including $Z$-band images from LRIS and WFC, and archival mid-infrared images from Spitzer/IRAC.

We recover S Ori 70 and the two faintest cluster member candidates from Caballero et al. (2007), and we find five red $I-J$ sources, with $J \sim 21 \mathrm{mag}$, located within 12 arcmin of the cluster centre. The near- and mid-infrared colours indicate that one of the sources, S Ori 73, is probably of T spectral type. If confirmed as a cluster member, it would be the least massive free-floating T type object detected in $\sigma$ Orionis, with $4_{-2}^{+3} M_{\text {Jup }}$. The four other sources appear to be L/T transition objects, but two are likely to be galaxies because of their strong mid-infrared excesses, similar to those of galaxies at $J \gtrsim 20.5$ mag. S Ori 72 and S Ori 73 are relatively close to the expected cluster sequence in the $J$ versus $J-[3.6]$ and $J-[4.5]$ colour-magnitude diagrams. S Ori 74 is located $11.8 \operatorname{arcsec}(\sim 4250$ AU) away from the solartype cluster star Mayrit 260182. From the effective search area 
Appendix A: Representation of individual fields in the IZJHK-bands
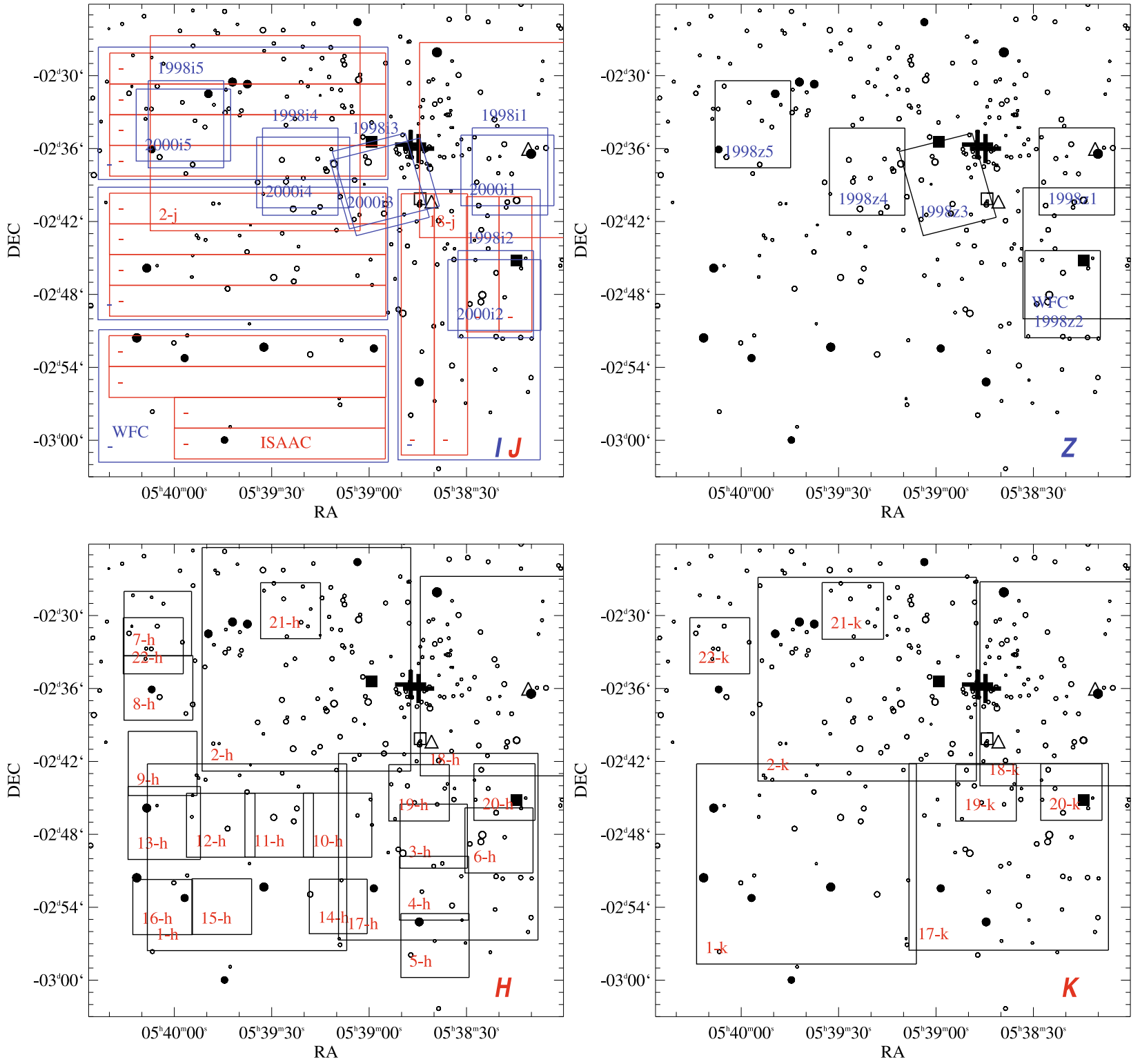

Fig. A.1. Individual fields used in the search: $I J$-bands (top left), Z-band (top right), $H$-band (bottom left), and $K$-band (bottom right). Symbols are defined as in Fig. 2.

of $\sim 790 \mathrm{arcmin}^{2}$ complete to $J=21.1 \mathrm{mag}$, we estimate there to be, after contaminant correction, between zero and two cluster members in the mass interval 6-4 $M_{\text {Jup }}$. The low number of candidates in this mass bin may be indicative of a turnover in the substellar mass function. Wider and deeper optical-to-infrared surveys are required to confirm whether this is the case, by constraining the mass function more tightly at lower masses.

Acknowledgements. We thank the referee Kevin Luhman. We thank Claire Halliday (A\&A language editor) and Terry Mahoney (IAC, Spain) for revising the English of the manuscript. We acknowledge Project No. 03065/PI/05 from the Fundación Séneca. Partially funded by the Spanish MEC under the Consolider-Ingenio 2010 Programme grant CSD2006-00070 (First Science with the GTC, http://www.iac.es/consolider-ingenio-gtc/). Based on observations made with ESO Telescopes at the La Silla or Paranal Observatories under programmes ID 068.C-0553(A) and 078.C-0402(A). Based on observations obtained at the Canada-France-Hawaii Telescope (CFHT), which is operated by the National Research Council of Canada, the Institut National des Sciences de l'Univers of the Centre National de la Recherche Scientifique of France, and the University of Hawaii. Based on observations collected at the German-Spanish Astronomical centre, Calar Alto, jointly operated by the Max-Planck-Institut für Astronomie Heidelberg and the Instituto de Astrofísica de Andalucía (CSIC). We thank Calar Alto Observatory for allocation of director's discretionary time to this programme. Based on observations made with the Isaac Newton Telescope (INT) and the William Herschel Telescope (WHT) operated on the island of La Palma by the Isaac Newton Group in the Spanish Observatorio del Roque de los Muchachos of the Instituto de Astrofísica de Canarias. This research has been supported by the Spanish Ministry of Science and Innovation (MICINN) under the grant AYA2007-67458. Some of the data presented herein were obtained at the W.M. Keck Observatory, which is operated as a scientific partnership among the California Institute of Technology, the University of California, and the National Aeronautics and Space Administration. The Observatory was made possible by the generous financial support of the W.M. Keck Foundation. The authors wish to recognise and acknowledge the very significant cultural role and reverence that the summit of Mauna Kea has always had within the indigenous Hawaiian community. We are most fortunate to have the opportunity to conduct observations from this mountain. This work is based in part on observations made with the Spitzer Space Telescope, which is operated by the Jet Propulsion Laboratory, 


\section{Appendix B: Finding charts}

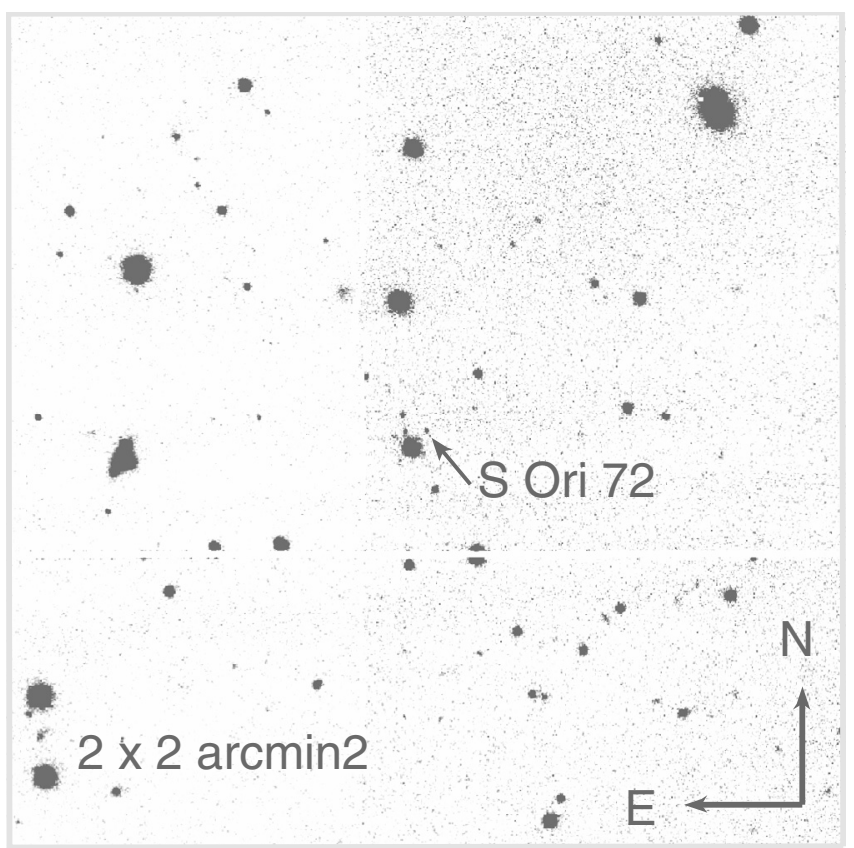

Fig. B.1. ISAAC $J$-band image of S Ori 72.

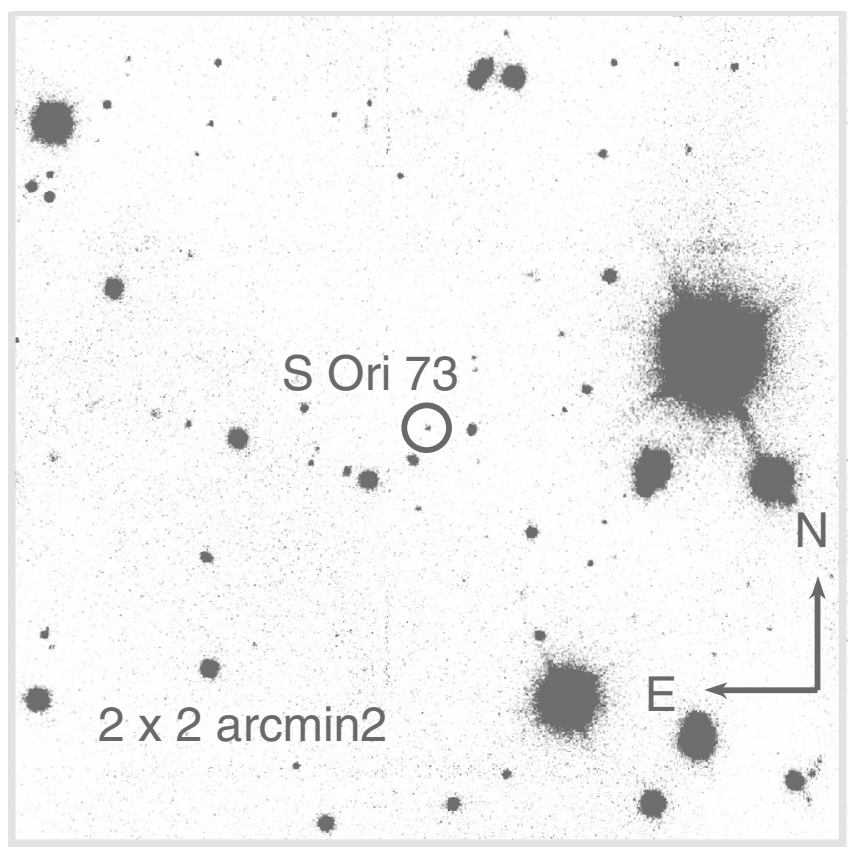

Fig. B.2. ISAAC $J$-band image of S Ori 73.

California Institute of Technology under a contract with NASA. IRAF is distributed by the National Optical Astronomy Observatories, which are operated by the Association of Universities for Research in Astronomy, Inc., under cooperative agreement with the National Science Foundation. This publication makes use of data products from the Two Micron All Sky Survey, which is a joint project of the University of Massachusetts and the Infrared Processing and Analysis centre/California Institute of Technology, funded by the National Aeronautics and Space Administration and the National Science Foundation. This research has made use of SAOImage DS9, developed by Smithsonian Astrophysical Observatory. This research has made use of the SIMBAD database, operated at CDS, Strasbourg, France.

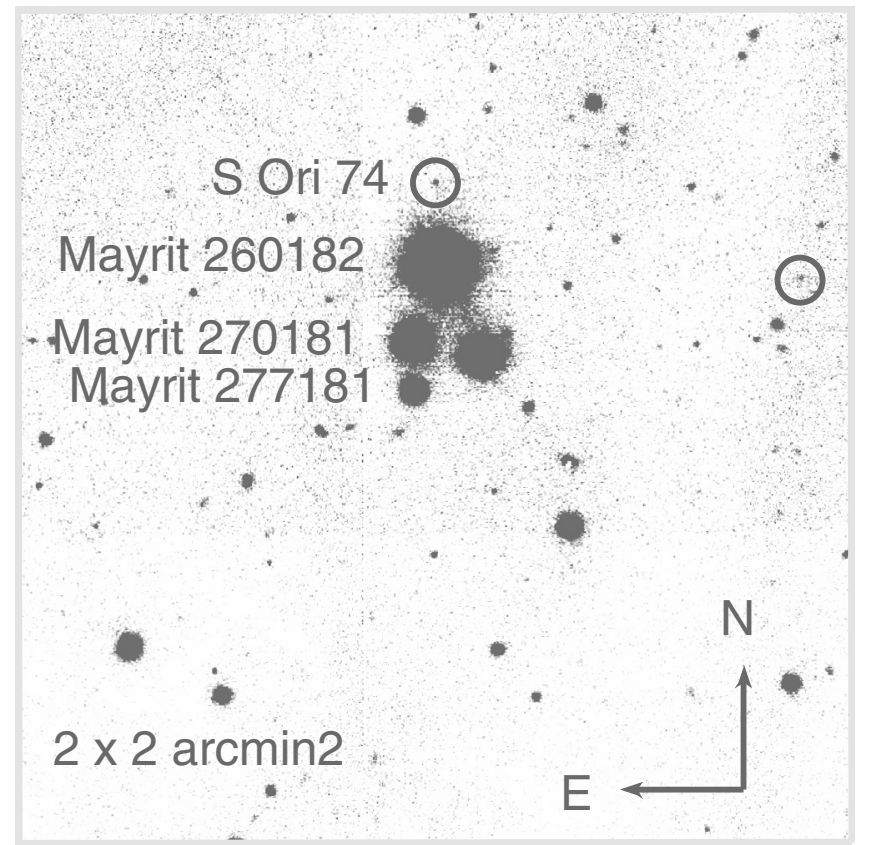

Fig. B.3. ISAAC $J$-band image of SOri 74 and S Ori J053840.8-024022 (unlabelled circle).

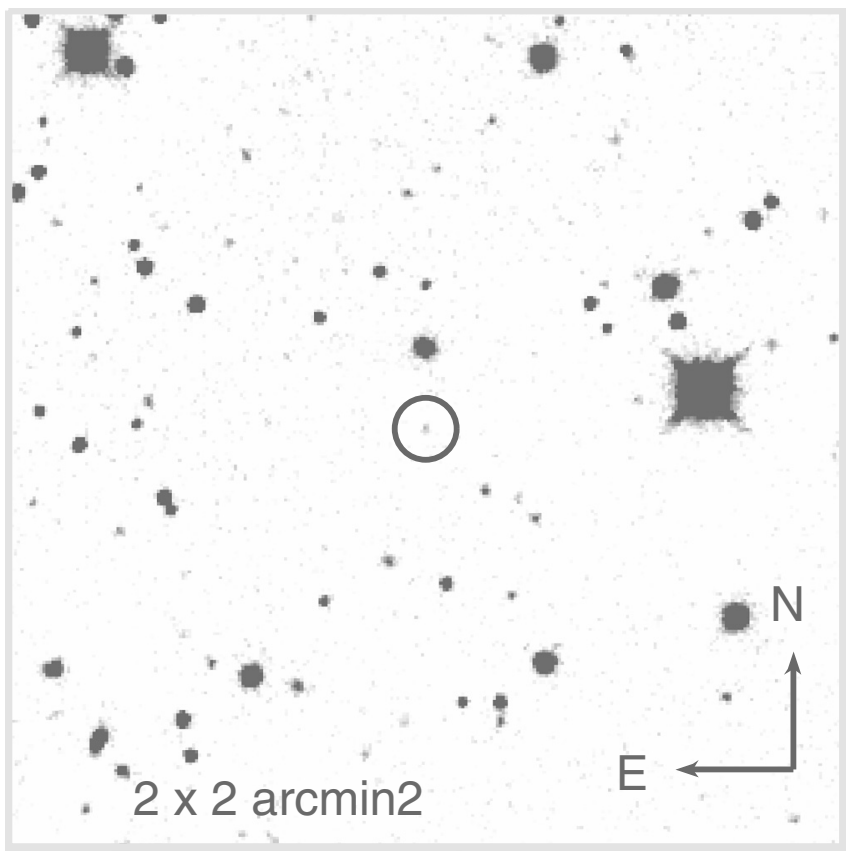

Fig. B.4. Omega2000 $H$-band image of S Ori J053811.0-023601.

\section{References}

Baraffe, I., Chabrier, G., Barman, T. S., Allard, F., \& Hauschildt, P. H. 2003, A\&A, 402, 701

Bate, M. R. 2005, MNRAS, 363, 363

Bate, M. R. 2009, MNRAS, 392, 590

Bate, M. R., \& Bonnell, I. A. 2005, MNRAS, 356, 1201

Beichman, C. A., Cutri, R., Jarrett, T., Stiening, R., \& Skrutskie, M. 2003, AJ, 125,2521

Béjar, V. J. S., Martín, E. L., Zapatero Osorio, M. R., et al. 2001, ApJ, 556, 830 Bihain, G., Rebolo, R., Béjar, V. J. S., et al. 2006, A\&A, 458, 805

Boss, A. P. 1997, Science, 276, 1836

Bouy, H., Huélamo, N., Martín, E. L., et al. 2009, A\&A, 493, 931 
Brown, A. G. A., de Geus, E. J., \& de Zeeuw, P. T. 1994, A\&A, 289, 101 Burgasser, A. J., Kirkpatrick, J. D., McGovern, M. R., et al. 2004, ApJ, 604, 827

Caballero, J. A. 2006, Ph.D. Thesis, Universidad de La Laguna, Spain

Caballero, J. A. 2007, A\&A, 466, 917

Caballero, J. A. 2008a, MNRAS, 383, 375

Caballero, J. A. 2008b, A\&A, 478, 667

Caballero, J. A. 2009, in The 15th Cambridge Workshop on Cool Stars, Stellar Systems, and the Sun, ed. E. Stempels, AIP Conf. Proc., 1094, 912

Caballero, J. A., Martín, E. L., Dobbie, P. D., \& Barrado y Navascués, D. 2006, A\&A, 460, 635

Caballero, J. A., Béjar, V. J. S., Rebolo, R., et al. 2007, A\&A, 470, 903

Caballero, J. A., Burgasser, A. J., \& Klement, R. 2008a, A\&A, 488, 181

Caballero, J. A., Valdivielso, L., Martín, E. L., et al. 2008b, A\&A, 491, 515

Carpenter, J. M. 2001, AJ, 121, 2851

Casali, M., Adamson, A., Alves de Oliveira, C., et al. 2007, A\&A, 467, 777

Chabrier, G., \& Baraffe, I. 2000, ARA\&A, 38, 337

Fortney, J. J., Marley, M. S., Saumon, D., \& Lodders, K. 2008, ApJ, 683, 1104

González-García, B. M., Zapatero Osorio, M. R., Béjar, V. J. S., et al. 2006, A\&A, 460, 799

González Hernández, J. I., Caballero, J. A., Rebolo, R., et al. 2008, A\&A, 490, 1135

Grazian, A., Fontana, A., de Santis, C., et al. 2006, A\&A, 449, 951

Hambly, N. C., Collins, R. S., Cross, N. J. G., et al. 2008, MNRAS, 384, 637

Hernández, J., Hartmann, L., Megeath, T., et al. 2007, ApJ, 662, 1067

Hester, J. J., Scowen, P. A., Sankrit, R., et al. 1996, AJ, 111, 2349

Hewett, P. C., Warren, S. J., Leggett, S. K., \& Hodgkin, S. T. 2006, MNRAS, 367,454

Hoyle, F. 1953, ApJ, 118, 513

Joye, W. A., \& Mandel, E. 2003, in Astronomical Data Analysis Software and Systems XII, ed. H. E. Payne, R. I. Jedrzejewski, \& R. N. Hook, PASPC, 295, 489

Landsman, W. B. 1993, in PASPC, Astronomical Data Analysis Software and Systems II, ed. R. J. Hanisch, R. J. V. Brissenden, \& J. Barnes, 52, 246

Larson, R. B. 1973, MNRAS, 161, 133

Lawrence, A., Warren, S. J., Almaini, O., et al. 2007, MNRAS, 379, 1599

Lodieu, N., Dobbie, P. D., Deacon, N. R., Venemans, B. P., \& Durant, M. 2009a, MNRAS, 395, 1631
Lodieu, N., Zapatero Osorio, M. R., Rebolo, R., Martín, E. L., \& Hambly, N. C. 2009b, A\&A, 505, 1115

Low, C., \& Lynden-Bell, D. 1976, MNRAS, 176, 367

Lucas, P. W., \& Roche, P. F. 2000, MNRAS, 314, 858

Luhman, K. L., Hernández, J., Downes, J. J., Hartmann, L., \& Briceño, C. 2008, ApJ, 688, 362

Martín, E. L., \& Zapatero Osorio, M. R. 2003, ApJ, 593, L113

Mayne, N. J., \& Naylor, T. 2008, MNRAS, 386, 261

Metchev, S. A., Kirkpatrick, J. D., Berriman, G. B., \& Looper, D. 2008, ApJ, 676,1281

Newberry, M. V. 1991, PASP, 103, 122

Padoan, P., \& Nordlund, Å. 2002, ApJ, 576, 870

Padoan, P., \& Nordlund, A. 2004, ApJ, 617, 559

Padoan, P., Nordlund, A., Kritsuk, A. G., Norman, M. L., \& Li, P. S. 2007, ApJ, 661, 972

Patten, B. M., Stauffer, J. R., Burrows, A., et al. 2006, ApJ, 651, 502

Rees, M. J. 1976, MNRAS, 176, 483

Scholz, A., \& Jayawardhana, R. 2008, ApJ, 672, L49

Sherry, W. H., Walter, F. M., Wolk, S. J., \& Adams, N. R. 2008, AJ, 135, 1616 Silk, J. 1977, ApJ, 214, 152

Skrutskie, M. F., Cutri, R. M., Stiening, R., et al. 2006, AJ, 131, 1163

Stamatellos, D., \& Whitworth, A. P. 2009, MNRAS, 392, 413

Stern, D., Eisenhardt, P., Gorjian, V., et al. 2005, ApJ, 631, 163

Tinney, C. G., Burgasser, A. J., \& Kirkpatrick, J. D. 2003, AJ, 126, 975

Veras, D., Crepp, J. R., \& Ford, E. B. 2009, ApJ, 696, 1600

Vrba, F. J., Henden, A. A., Luginbuhl, C. B., et al. 2004, AJ, 127, 2948

Whitworth, A. P., \& Goodwin, S. P. 2005, Astron. Nachr., 326, 899

Whitworth, A. P., \& Stamatellos, D. 2006, A\&A, 458, 817

Whitworth, A. P., \& Zinnecker, H. 2004, A\&A, 427, 299

Zapatero Osorio, M. R., Béjar, V. J. S., Martín, E. L., et al. 2000, Science, 290, 103

Zapatero Osorio, M. R., Béjar, V. J. S., Martín, E. L., et al. 2002a, ApJ, 578, 536 Zapatero Osorio, M. R., Béjar, V. J. S., Pavlenko, Y., et al. 2002b, A\&A, 384, 937

Zapatero Osorio, M. R., Caballero, J. A., Béjar, V. J. S., et al. 2007, A\&A, 472, L9

Zapatero Osorio, M. R., Béjar, V. J. S., Bihain, G., et al. 2008, A\&A, 477, 895 Zhang, Z. H., Pokorny, R. S., Jones, H. R. A., et al. 2009, A\&A, 497, 619 